\title{
Effects of Non-invasive Neurostimulation on Autism Spectrum Disorder: A Systematic Review
}

\author{
Ali Khaleghi', Hadi Zarafshan', Safa Rafiei Vand ${ }^{2}$, Mohammad Reza Mohammadi \\ ${ }^{1}$ Psychiatry and Psychology Research Center, Roozbeh Hospital, Tehran University of Medical Sciences, ${ }^{2}$ Faculty of Biomedical Engineering, \\ Amirkabir University of Technology, Tehran, Iran
}

\begin{abstract}
Autism spectrum disorder (ASD) is a complex neurodevelopmental disorder characterized by major impairments in social communication, stereotyped and ritualistic behaviors and deficits in sensory reactivity. Recently, noninvasive brain stimulation (NIBS) methods, namely transcranial direct current stimulation (tDCS) and transcranial magnetic stimulation (TMS), have been examined as possible new therapeutic options for modifying the pathological neuroplasticity involved in neuropsychiatric disorders including ASD. Therefore, we conducted a systematic review on the therapeutic uses of tDCS and repetitive TMS (rTMS) in ASD patients. A systematic search was performed on Scopus, Web of Science, PubMed, Cochrane and Embase. Original articles reporting the use of tDCS or rTMS to treat ASD were screened and studied by two researchers independently based on PRISMA guidelines. We found 32 eligible studies including 8 tDCS reports, 23 rTMS reports and one report with both tDCS and rTMS. These studies comprised 6 case-reports, 9 non-controlled trials and 17 controlled trials which assessed NIBS effects on the three cognitive, behavioral and biological dimensions in ASD. Existing evidence demonstrates that NIBS methods could be helpful for treating some dimensions of ASD such as repetitive behavior, sociability or some aspects of executive and cognitive functions. However, such evidence should be regarded with care because of the quality of original researches and serious publication bias as well as the heterogeneity of data. Further randomized, double-blind, sham-controlled trials with appropriate follow-up periods should be designed to assess the efficacy of NIBS methods for ASD treatment.
\end{abstract}

KEY WORDS: Autism; Non-invasive neurostimulation; Transcranial direct current stimulation; Transcranial magnetic stimulation; Brain.

\section{INTRODUCTION}

Autism spectrum disorder (ASD) is a complex neurodevelopmental disorder characterized by major impairments in social communication and interaction, stereotyped and ritualistic behaviors and deficits in sensory reactivity [1]. Recently, its prevalence has grown dramatically around the world and is reported as $1 \%$ in the Diagnostic and Statistical Manual of Mental Disorders 5th edition (DSM-5) [2-4]. People with autism show deficits in several domains such as cognition, memory, attention,

Received: February 24, 2020 / Revised: June 8, 2020

Accepted: June 10, 2020

Address for correspondence: Ali Khaleghi

Psychiatry and Psychology Research Center, Roozbeh Hospital, Tehran University of Medical Sciences, South Kargar Ave., Tehran 1333795914, Iran

E-mail: alikhaleghi_bme84@yahoo.com

ORCID: https://orcid.org/0000-0002-9035-7075 emotion recognition and regulation, and social skills [5].

Although the etiology and pathophysiology of ASD are not conclusively clear, neuroimaging studies have reported abnormalities in patterns of brain perfusion [6], regional brain volumes [7], excitatory/inhibitory neurotransmission and synaptic plasticity [8], and neural biochemical characteristics of ASD [9]. These abnormalities are not limited to a single brain region; rather they are the result of a breakdown in the integration and functioning of long-range neural circuits. Some neurophysiological findings that may be underlying pathophysiological causes of symptoms associated with ASD include the larger volume of right brain structures associated with social function and language [10], hypoactivation of specific brain regions (such as amygdala) related to social cognition and face processing [11], abnormal synaptic development and aberrant reduction of cortical plasticity [12], mirror

(c) This is an Open-Access article distributed under the terms of the Creative Commons Attribution Non-Commercial License (http://creativecommons.org/licenses/by-nc/4.0) which permits unrestricted non-commercial use, distribution, and reproduction in any medium, provided the original work is properly cited. 
neuron dysfunction [13], and decreased inhibitory function in the GABAergic interneurons due to deficits in the peripheral compartment of the minicolumns and aberrant increase in the excitation to inhibition ratio in the cortical structure [14].

Different intervention approaches are used for people with autism. Most of these interventions are based on the behavioral approach and, to some extent, on the cognitive/developmental approach. Today, much attention is being paid to the use of devices and technologies in the treatment of autism. In the past decade, noninvasive brain stimulation (NIBS) methods, namely transcranial direct current stimulation (tDCS) and transcranial magnetic stimulation (TMS), have been examined as possible new therapeutic options for modification of the pathological neuroplasticity (or even plasticity induction) involved in neuropsychiatric disorders including ASD. Previous studies have shown that plasticity induction or modification in the human central nervous system with NIBS has various functional effects on learning, working memory and cognitive processes $[15,16]$. So far, different stimulation protocols have been proposed to induce alterations in cortical excitability. tDCS is one of the NIBS protocols which induces plasticity by alteration of membrane potentials, which modify spontaneous discharge rates using a subthreshold polarity-dependent electrical stimulation [17]. This leads to reduced or enhanced neuronal excitability during stimulation depending on the polarity and arrangement of anode and cathode electrodes. In fact, tDCS modulates cortical excitability by applying a low amplitude direct current (0.5-2 mA) through scalp electrodes [18]. In general, cortical excitability is reduced under the cathode and enhanced under the anode electrode. The tDCS effect outlasts the stimulation depending on the intensity and duration of current application. However, weekly repeated multisession tDCS may result in cumulative effects of stimulation on neural activities and may prolong its effects on behavioral outcomes [19]. It is thought that the short-term effects of tDCS occur by depolarization of membrane potentials at the resting-state through non-synaptic mechanisms [20], and its long-term effects occur by $\mathrm{N}$-methyl-D-aspartate-dependent mechanisms [21].

TMS is a major noninvasive neuromodulation technique that uses electromagnetic induction based on Faraday's principle to generate transient, localized, orthogonal electric fields in the brain cortex. In this approach, the magnetic field penetrates the skull, which is a highly resistant structure, and the resulting electric field generates secondary currents in an inner structure of the brain with a low depolarization threshold. This results in depolarization and firing of local neurons (i.e., neuronal activation) $[22,23]$. Repetitive TMS (rTMS) delivers a series of short magnetic pulses over a specified brain region, usually at a frequency range of $0.5-20 \mathrm{~Hz}$. At low frequencies (i.e., below $5 \mathrm{~Hz}$ ), rTMS leads to long-term suppression of brain excitability by mechanisms related to depression; whereas, at high frequencies (i.e., above $5 \mathrm{~Hz}$ ), rTMS typically results in long-term facilitation of brain excitability by mechanisms associated with long-term potentiation $[22,24]$. However, these effects are subject to interpersonal variability. One alternative form of rTMS is theta burst stimulation (TBS) which is designed to deliver three $50 \mathrm{~Hz}$ pulses over a chosen brain region repeated at $200 \mathrm{~ms}$ intervals. Intermittent TBS (iTBS) results in facilitation of cortical excitability, while continuous TBS (cTBS) has inhibitory effects on the cortex [25]. The effects of TBS are longer and more prominent than those induced by conventional rTMS [15]. All these effects outlast stimulation depending on the state of the stimulated brain region as well as the duration and magnitude of stimulation.

In 2008, the U.S. Food and Drug Administration (FDA) approved rTMS as a treatment to relieve mild symptoms associated with treatment-resistant depression in patients who have not found alleviation from antidepressant medication [26]. Furthermore, various studies suggest tDCS may be a useful tool to treat neuropsychiatric conditions. These studies have also shown cognitive improvement in some psychiatric conditions after tDCS interventions [27]. However, tDCS is not currently an FDA-approved treatment. Moreover, many studies in recent years have provided substantial evidence that both rTMS and tDCS are reasonably safe and well tolerated in human application when performed according to the recommended safety guidelines $[28,29]$. Therefore, these two NIBS techniques have attracted particular interest as potential treatment tools in ASD in the last decade. Although in recent reviews published in 2016 and 2018, Oberman et al. [30] and Barahona-Corrêa et al. [31] have well discussed the rTMS therapeutic effects on ASD symptoms, they ignored tDCS as a very important technique in NIBS. Thus, we attempted to provide a comprehensive overview of therapeutic effects resulting from both neurostimulation methods 
(tDCS and rTMS) on ASD. For this purpose, we conducted a systematic review of the literature for published original papers on the therapeutic uses of tDCS and rTMS in patients with ASD.

\section{SEARCH STRATEGY}

Our paper focuses on English language articles reporting the effect of neurostimulation interventions on human subjects with autism spectrum disorder who were diagnosed based on a valid method (i.e., clinical diagnosis based on the DSM or International Classification of Diseases criteria, or specific diagnostic tools). We did not consider any limitations regarding the study design, publication time, and age or sex of participants.

Our review was based on the Preferred Reporting Items for Systematic reviews and Meta-Analyses for Protocols
2015 (PRISMA-P 2015) guidelines. We searched MEDLINE/ PubMed, ISI Web of Science, Scopus, CENTRAL Cochrane and Embase databases up to April 2018 using keywords relevant to autism and neurostimulation interventions. Search terms indicated the diagnoses of interest (Autism Spectrum Disorder, Autism, Asperger) and the interventions of interest (Noninvasive Brain Stimulation, Transcranial, tDCS, transcranial alternating current stimulation [tACS], TMS, rTMS, TBS). After removing duplicates, two members of the research team separately screened articles based on their title and abstract and selected relevant articles based on the research question. In cases of disagreement, the decision was made based on the opinion of a third member in the research team. We also screened the reference list of selected articles to find any additional original reports. After that, we studied the full text of eligible articles to retrieve relevant data.

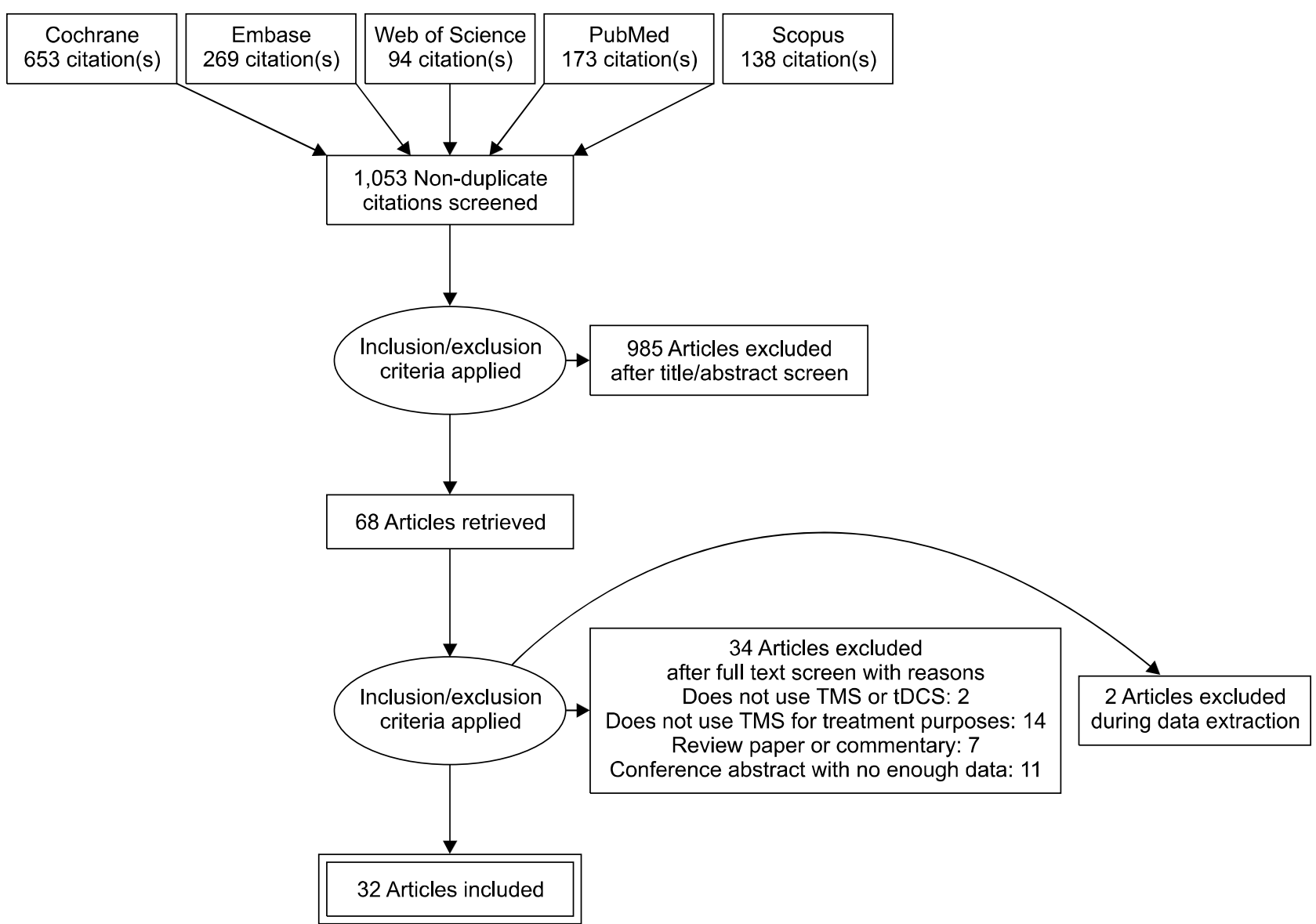

Fig. 1. Flow diagram and process of identification, screening and assessing eligibility of studies on the noninvasive brain stimulation effects on autism spectrum disorder.

tDCS, transcranial direct current stimulation; TMS, transcranial magnetic stimulation. 


\section{DATA EXTRACTION}

According to PRISMA guidelines, two researchers independently retrieved the relevant data from eligible articles including author's name, publication year, study design, number of participants, sex, mean age, type of stimulation, brain target, stimulation parameters, stimulation schedule, outcome measures, clinical outcomes, follow-up duration and additional information. We also evaluated papers for publication bias according to Cochrane guidelines [32].

\section{SYNTHESIZED FINDINGS}

The systematic search disclosed 1,053 unique studies. The screening step for finding those that conformed to the eligibility criteria led to the identification of 32 studies using rTMS and tDCS for therapeutic purposes in individuals with ASD. Figure 1 shows a schematic overview of the study selection process. Eight studies exclusively utilized tDCS as the therapeutic tool [33-40], 23 studies utilized solely rTMS as the therapeutic tool [41-63], and one study used both rTMS and tDCS to evaluate their therapeutic effects on ASD [64]. Six of the included articles were case-reports $[35,40,43,47,49,53]$, nine were non-controlled trials $[36,37,41,46,55,57,61,62,64]$, and the remaining 17 were controlled trials $[33,34,38,39,42,44,45$, $48,50-52,54,56,58-60,63]$. Eleven of the controlled studies used a sham group as the control $[33,34,38,39,42,48,50$ $52,54,63]$ and the remaining six compared neuromodulated patients with waitlist controls. Totally, 467 patients with ASD (mean age of 16.19) were treated by neuromodulation techniques in all included studies (383 patients received rTMS and 84 patients received tDCS). Nine studies recruited adult subjects with ASD [36,38,39,43, 48,49,51-53], and the remaining studies focused on children and adolescents with ASD. Subjects covered almost the entire autistic spectrum including high function, low function and Asperger with and without verbal and cognitive impairments. Tables 1 to 4 and Figure 2 have summarized the characteristics, technical parameters and outcomes of the included studies.

There was a large variability between studies regarding the stimulation protocols. Some studies applied singlesession stimulations, while some adopted multisession protocols of stimulation. In the tDCS technique, six stud- ies applied unilateral anodal or cathodal stimulations of dorsolateral prefrontal cortex (DLPFC; right and left), right temporoparietal junction and supplementary motor area (SMA); whereas two studies applied bilateral stimulation of DLPFC. In these studies, the currents of $0.4,1,1.5$ and $2 \mathrm{~mA}$ were delivered to the brain via 25 and $35 \mathrm{~cm} 2$ electrodes for different durations (20, 30, 40 and 85 minutes). In the rTMS technique, two studies adopted the TBS protocols, one study adopted the pico-Tesla TMS (pT-TMS) protocol, and the remaining 21 studies adopted more conventional rTMS approaches. In more conventional rTMS methods, as in tDCS, most studies delivered stimulation to the DLPFC, either unilaterally or bilaterally, using stimulation frequencies of $0.5-1 \mathrm{~Hz}$. Three studies delivered multisession stimulation to the bilateral medial prefrontal cortex (mPFC) with the stimulation frequency of $5 \mathrm{~Hz}$. One study delivered a stimulation of $1 \mathrm{~Hz}$ rTMS to the pars opercularis and pars triangularis of the inferior frontal gyrus bilaterally, while another study delivered a stimulation of $1 \mathrm{~Hz}$ rTMS to the SMA and left primary motor cortex (PMC). Moreover, a study targeted the premotor cortex, either unilaterally or bilaterally, using $1 \mathrm{~Hz}$ (low frequency stimulation) and $8 \mathrm{~Hz}$ (high frequency stimulation) rTMS. Also, two other studies applied $1 \mathrm{~Hz}$ rTMS and $20 \mathrm{~Hz}$ rTMS to the motor cortex. In TBS protocols, researchers stimulated bilateral DLPFC, right DLPFC and bilateral posterior superior temporal sulcus (pSTS) with iTBS. At last, pT-TMS trial delivered stimulation to the frontal cortex, vertex, bilateral temporal areas, bilateral parietal areas and occipital cortex with stimulation frequencies of $8-13 \mathrm{~Hz}$. In both techniques, treatment schedules and the number of stimulation sessions varied widely among the studies. A limited number of studies adopted neuronavigation approaches to guide stimulation of the brain area of interest. In rTMS studies, a research group used $\mathrm{H}$-coil, another research group used a customized helmet containing 122 coils (pT-TMS trial), and all remaining rTMS studies utilized conventional figure of eight coils. Furthermore, there was a large variability between studies regarding the outcome measures. Overall, the included studies reported over 30 outcome measures. Based on the reported results, we can categorize these outcome measures into the three domains (cognitive, behavioral and biological). Below, the main results of included studies are summarized per domain. 


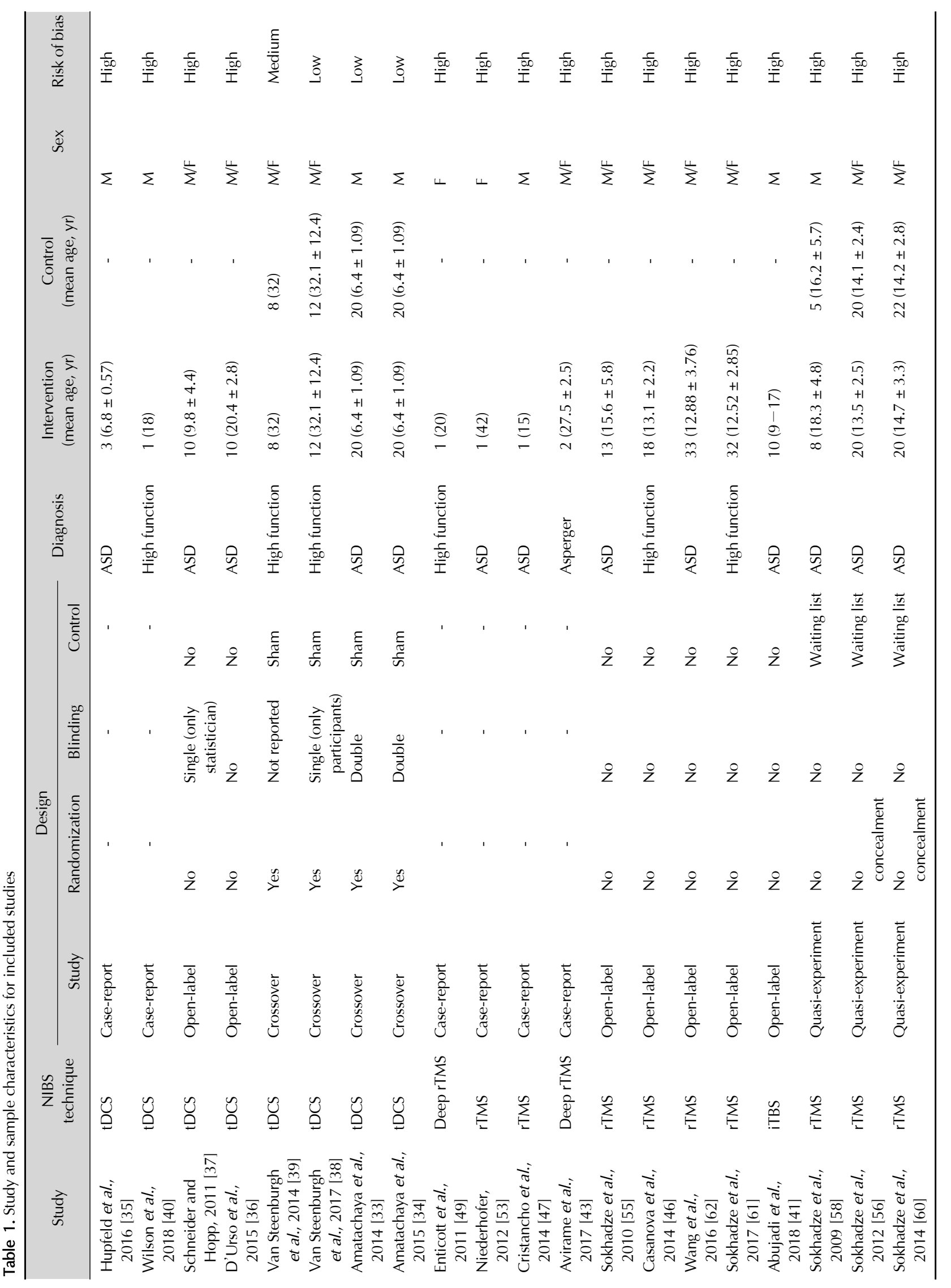




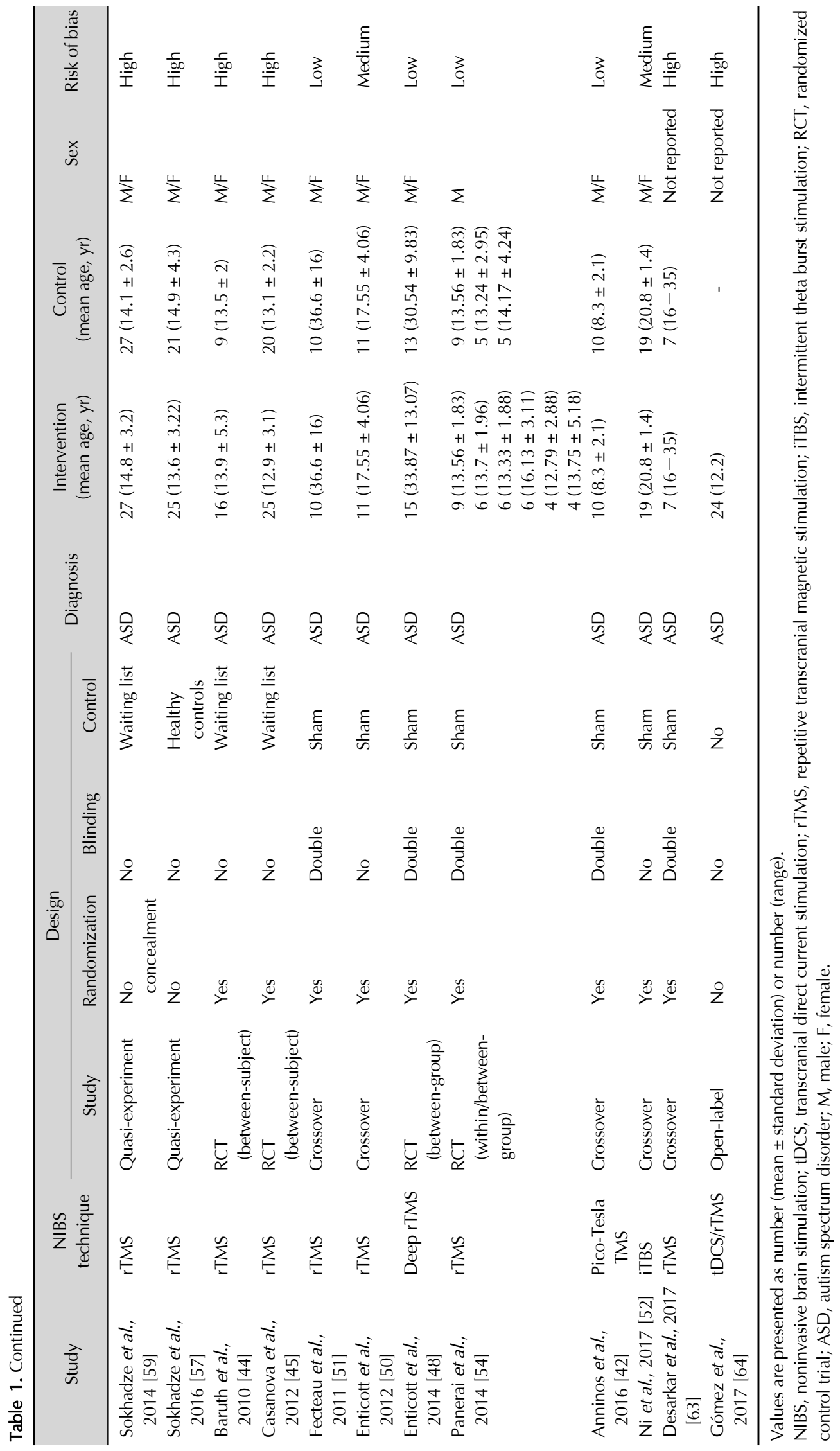




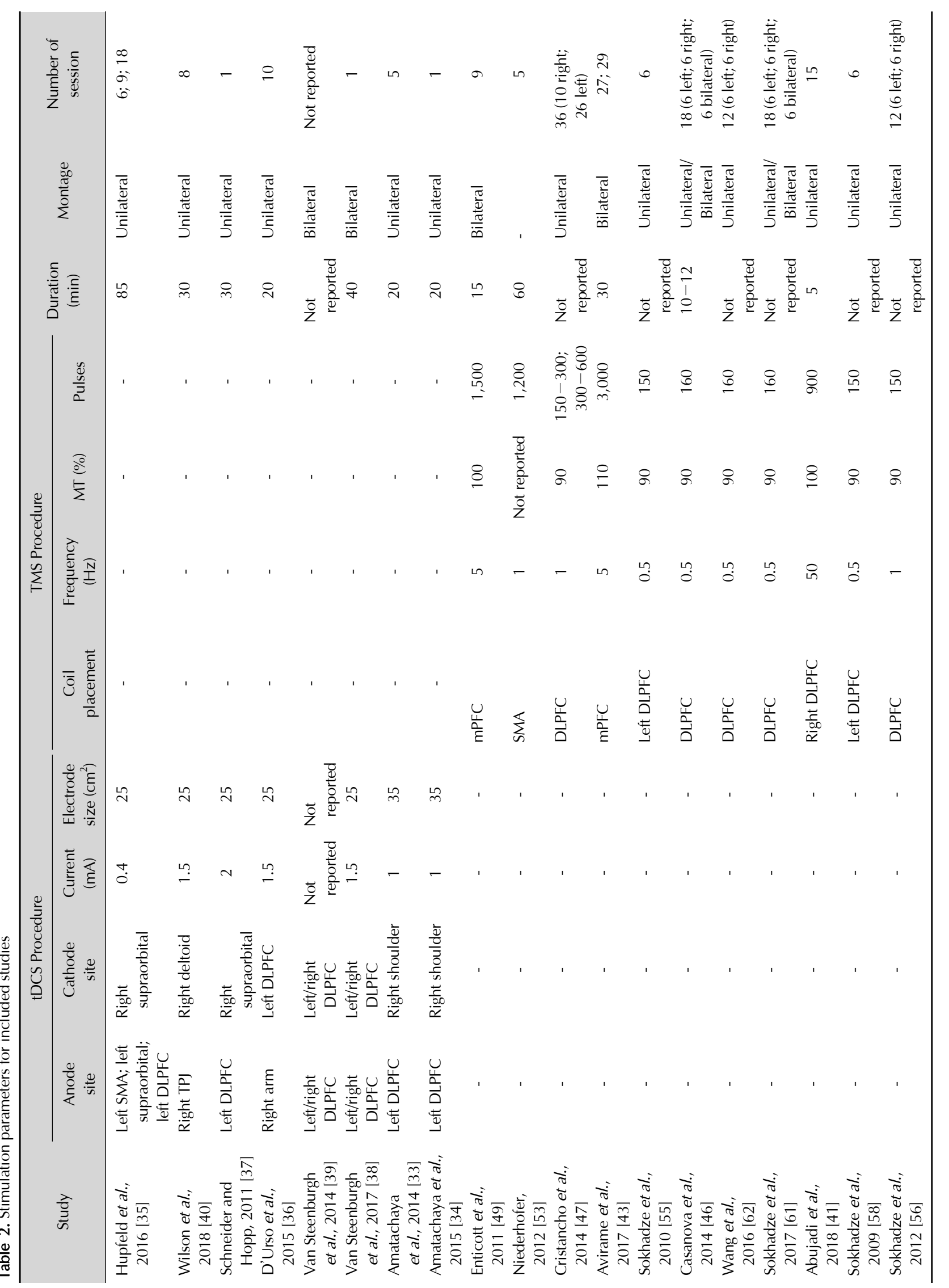




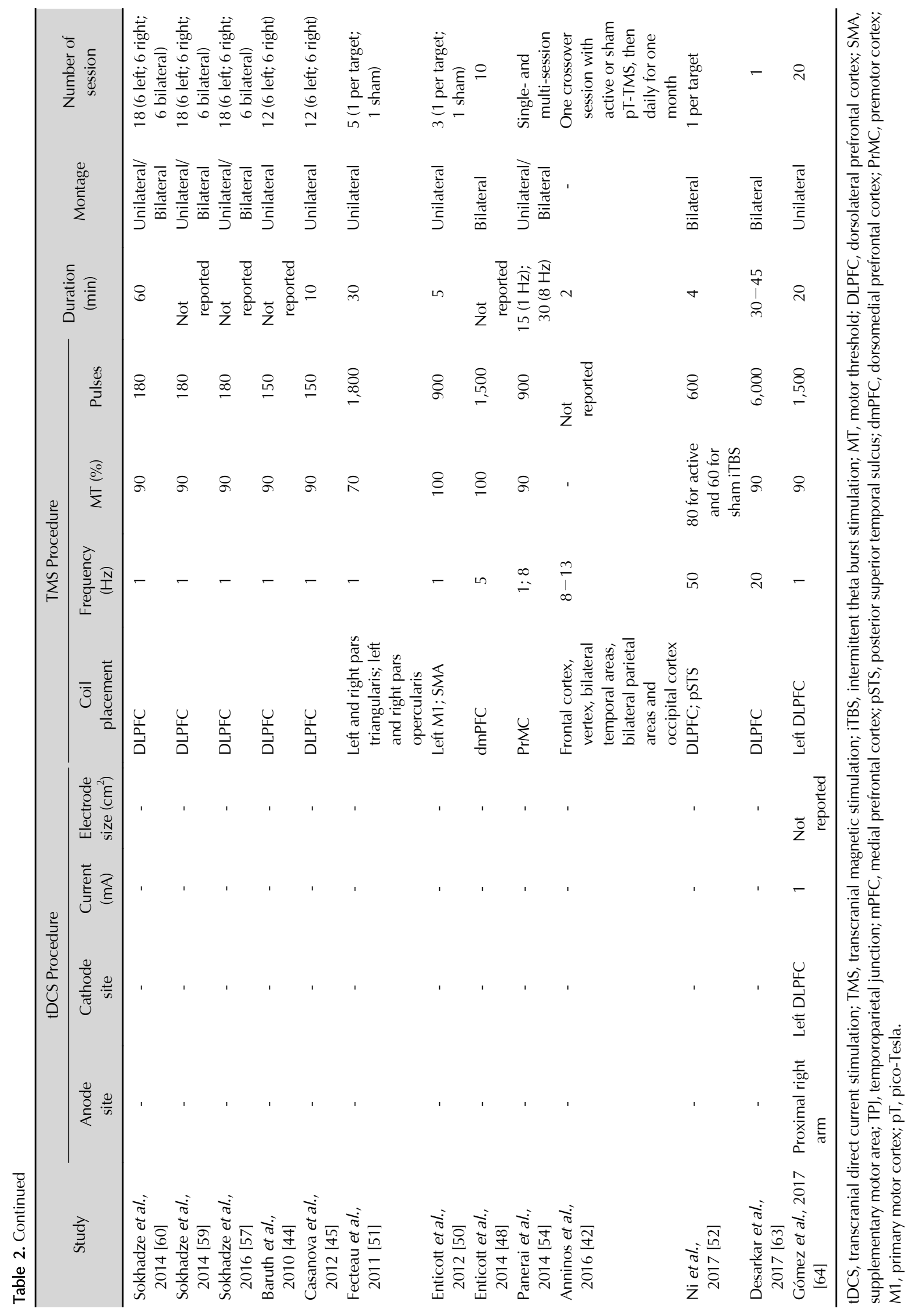




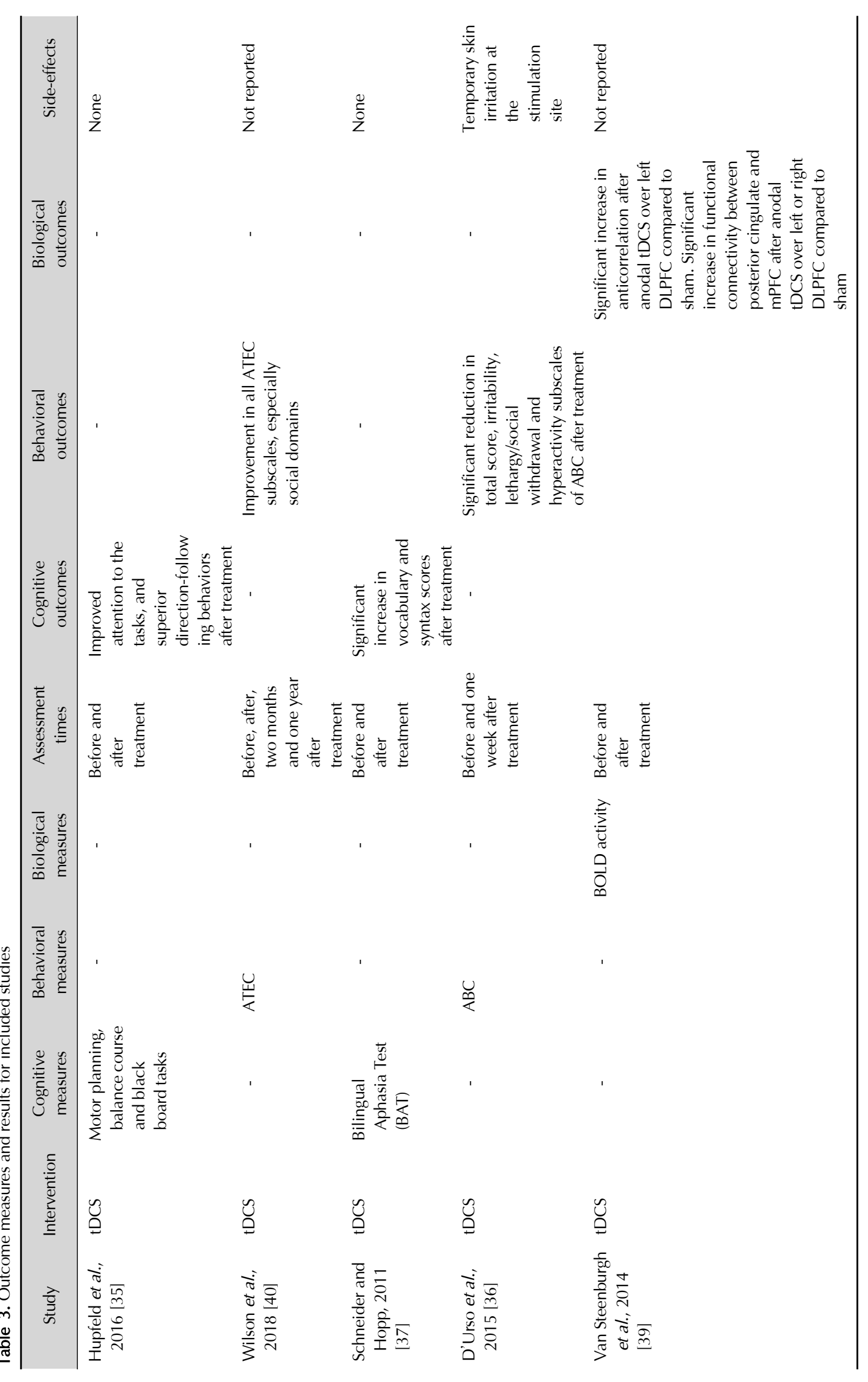




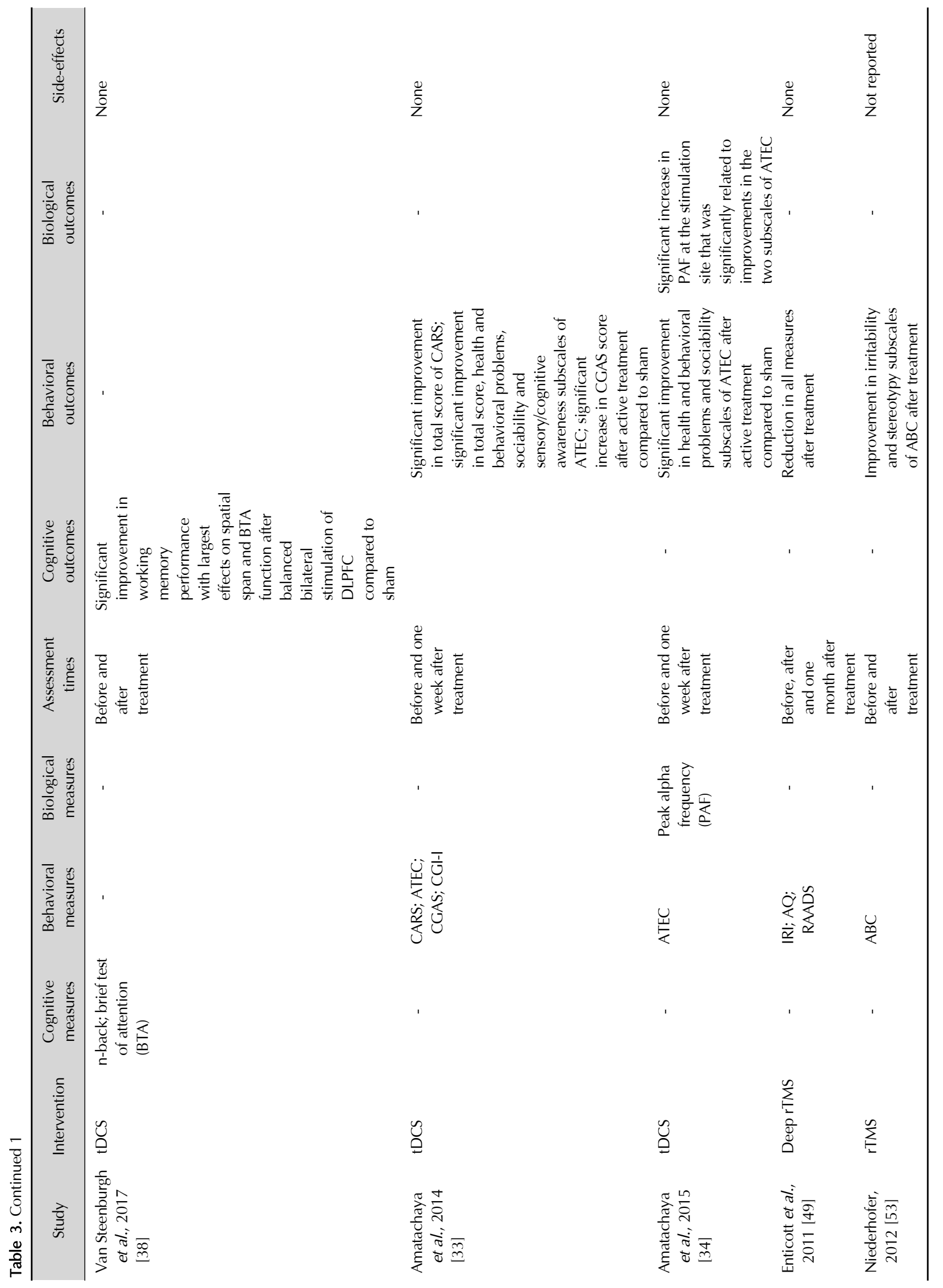




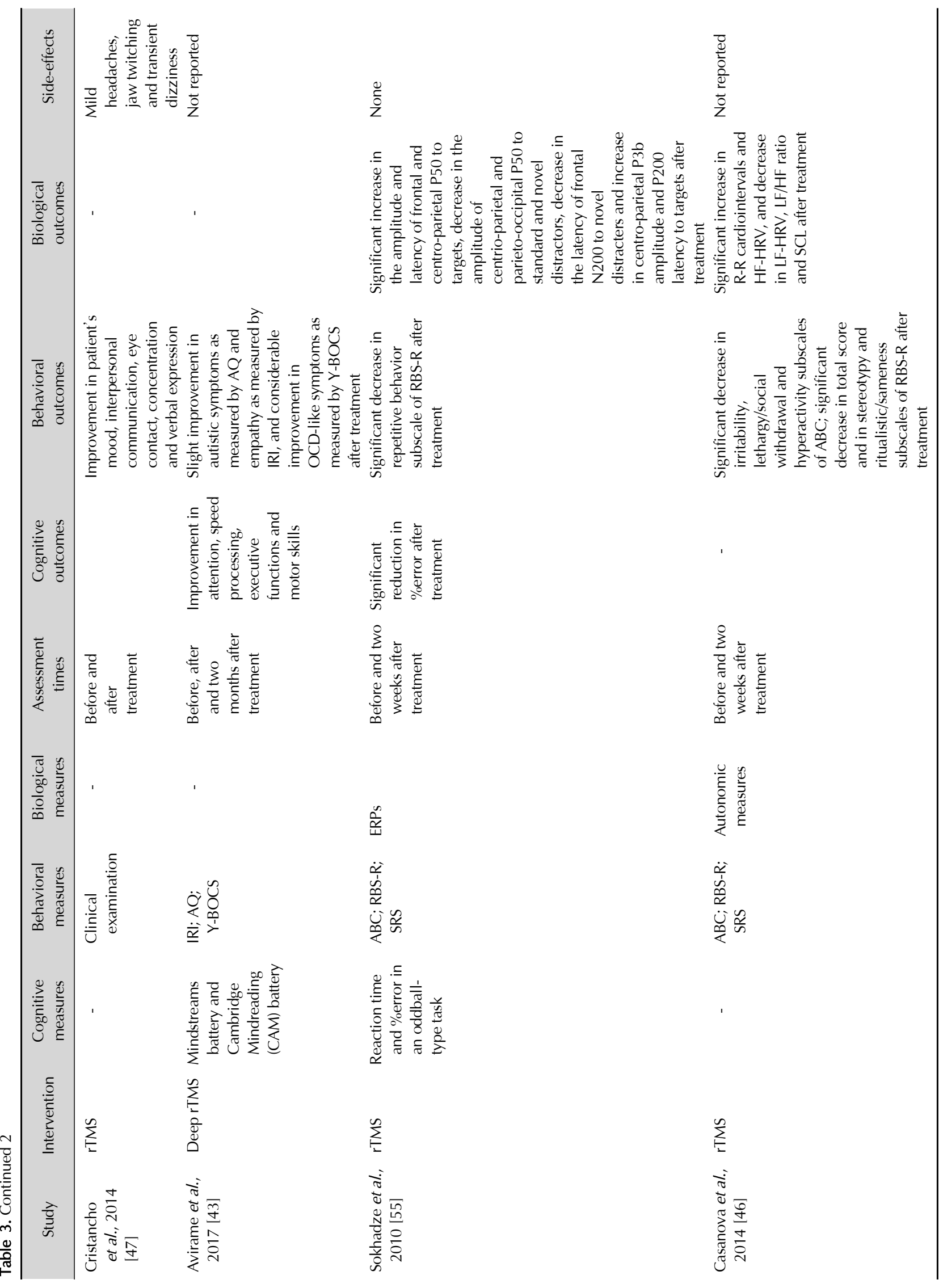




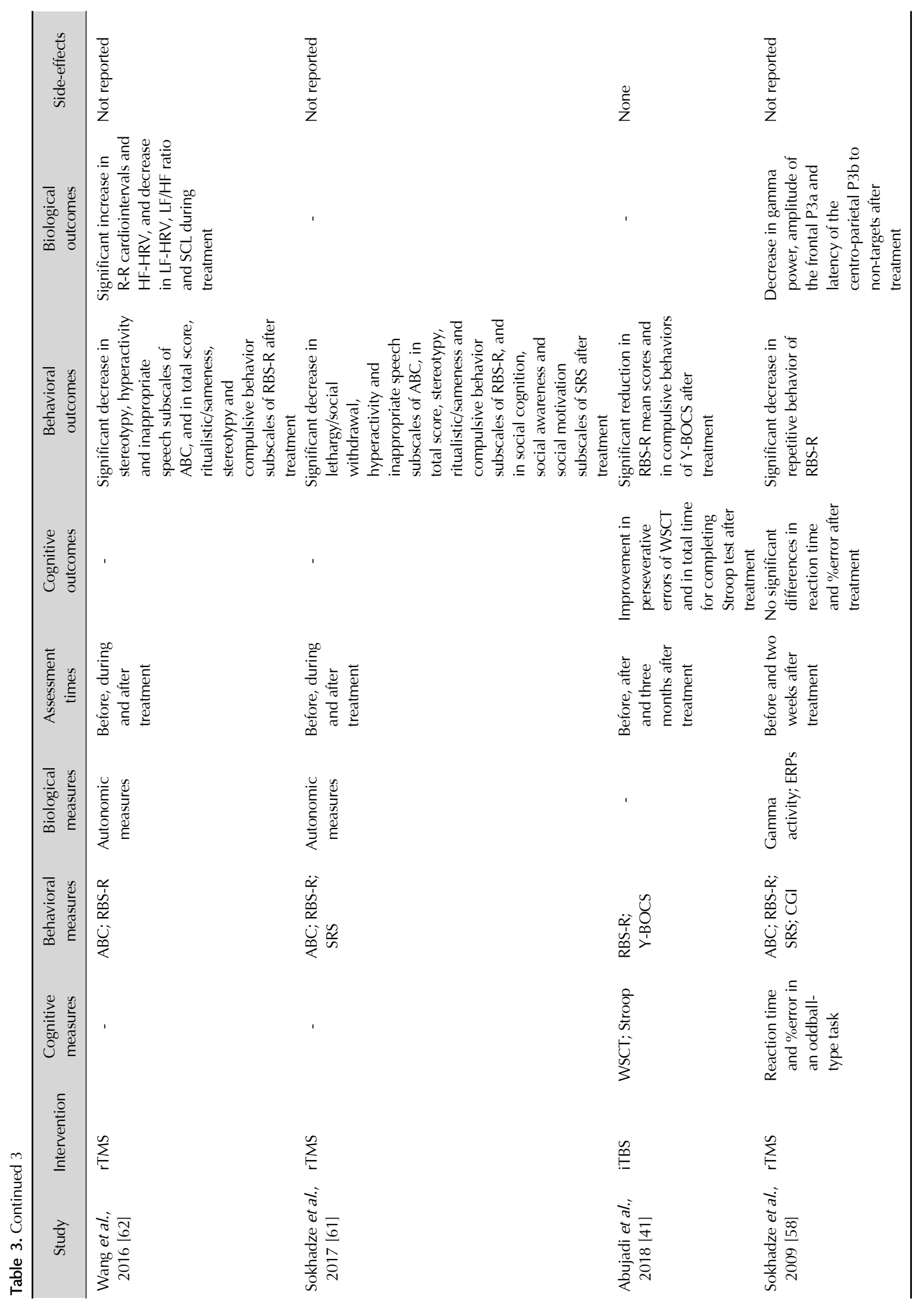




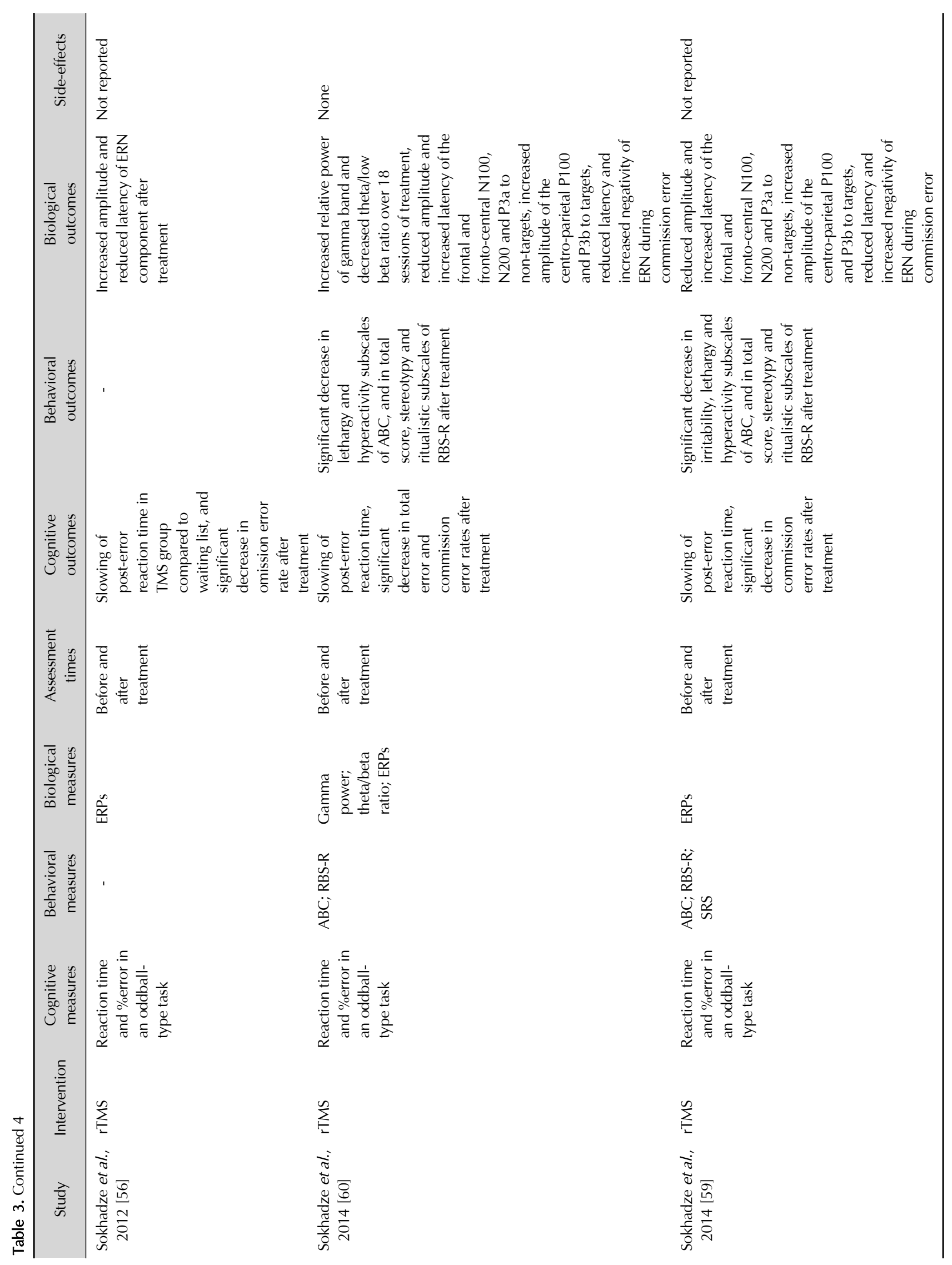




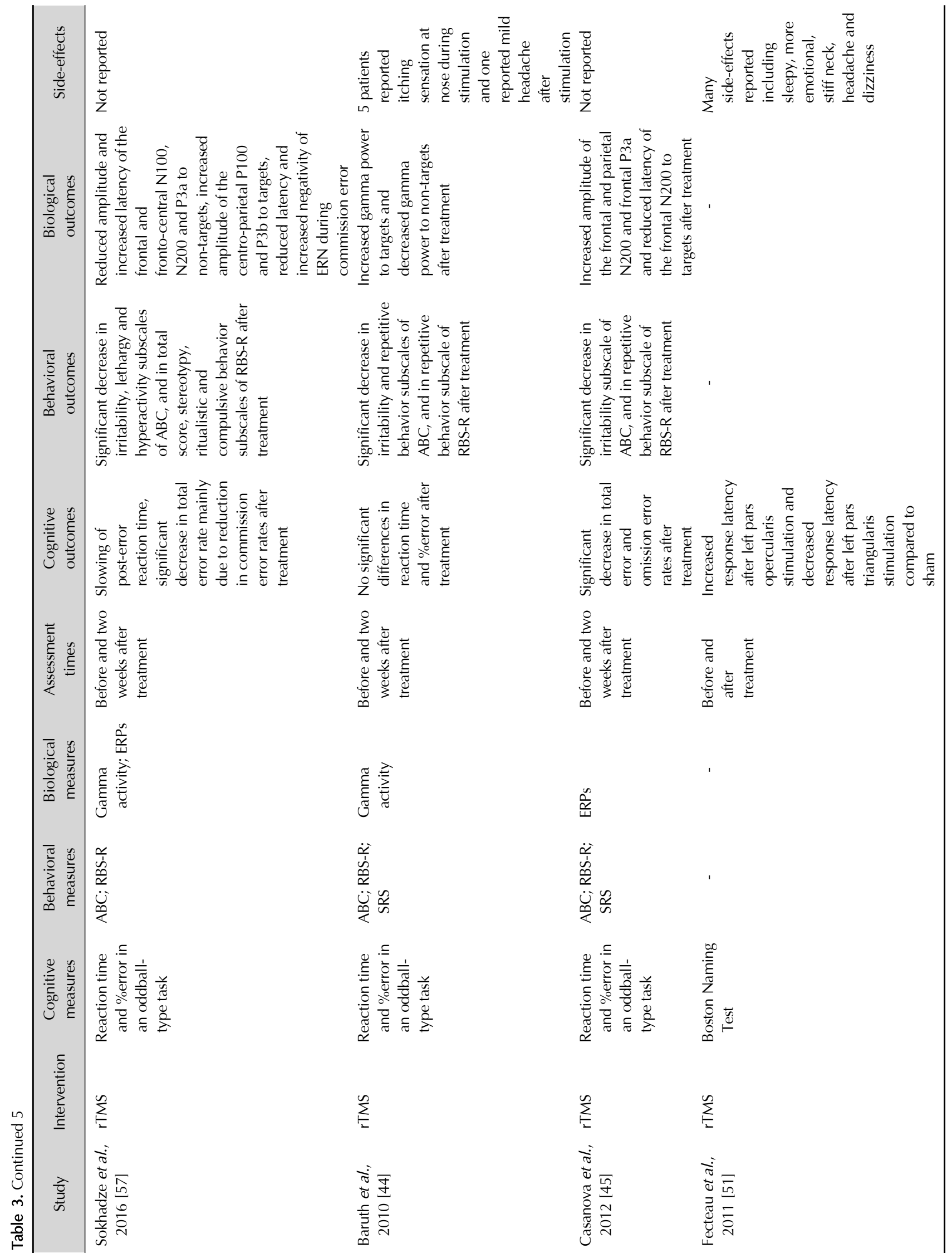




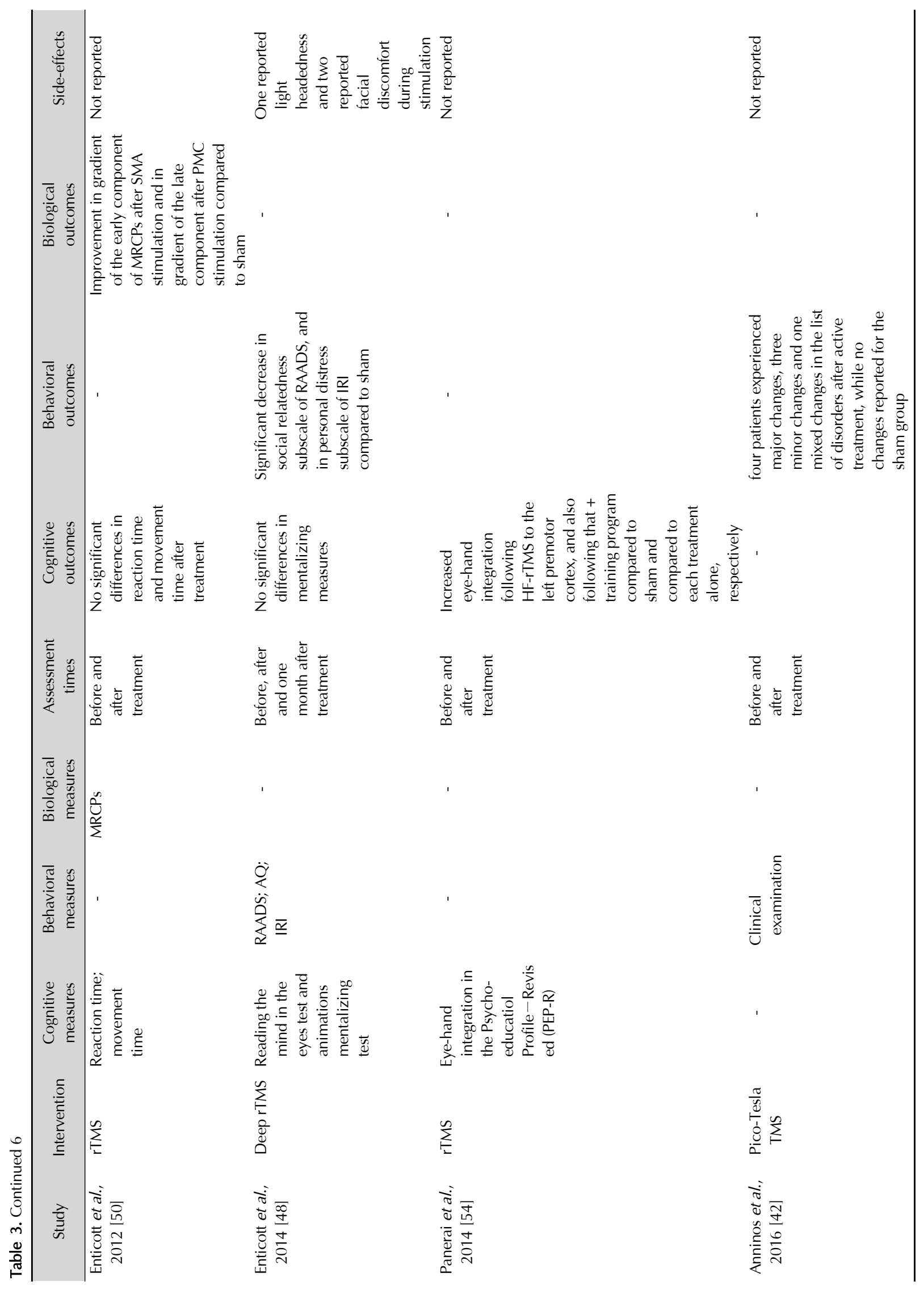




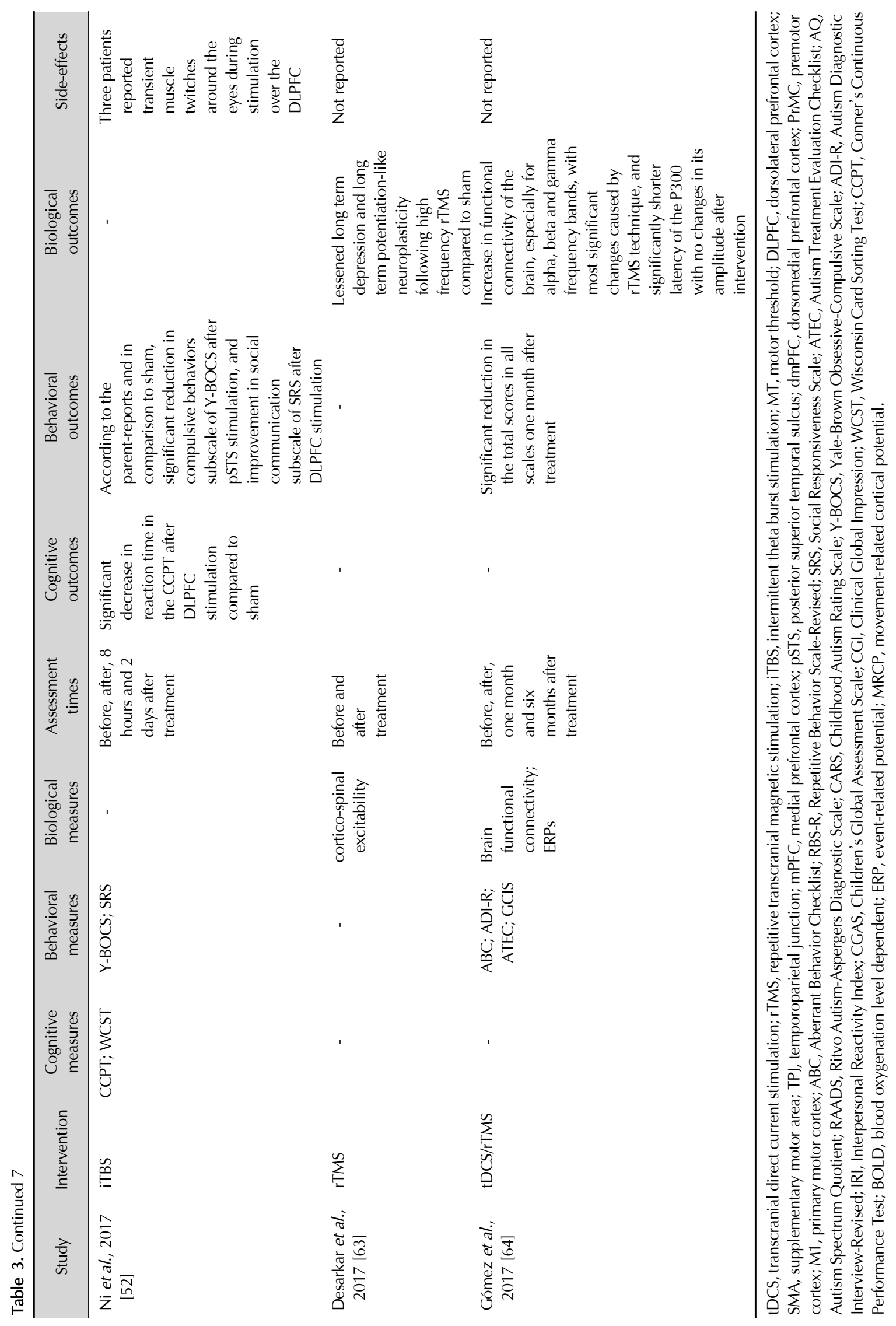




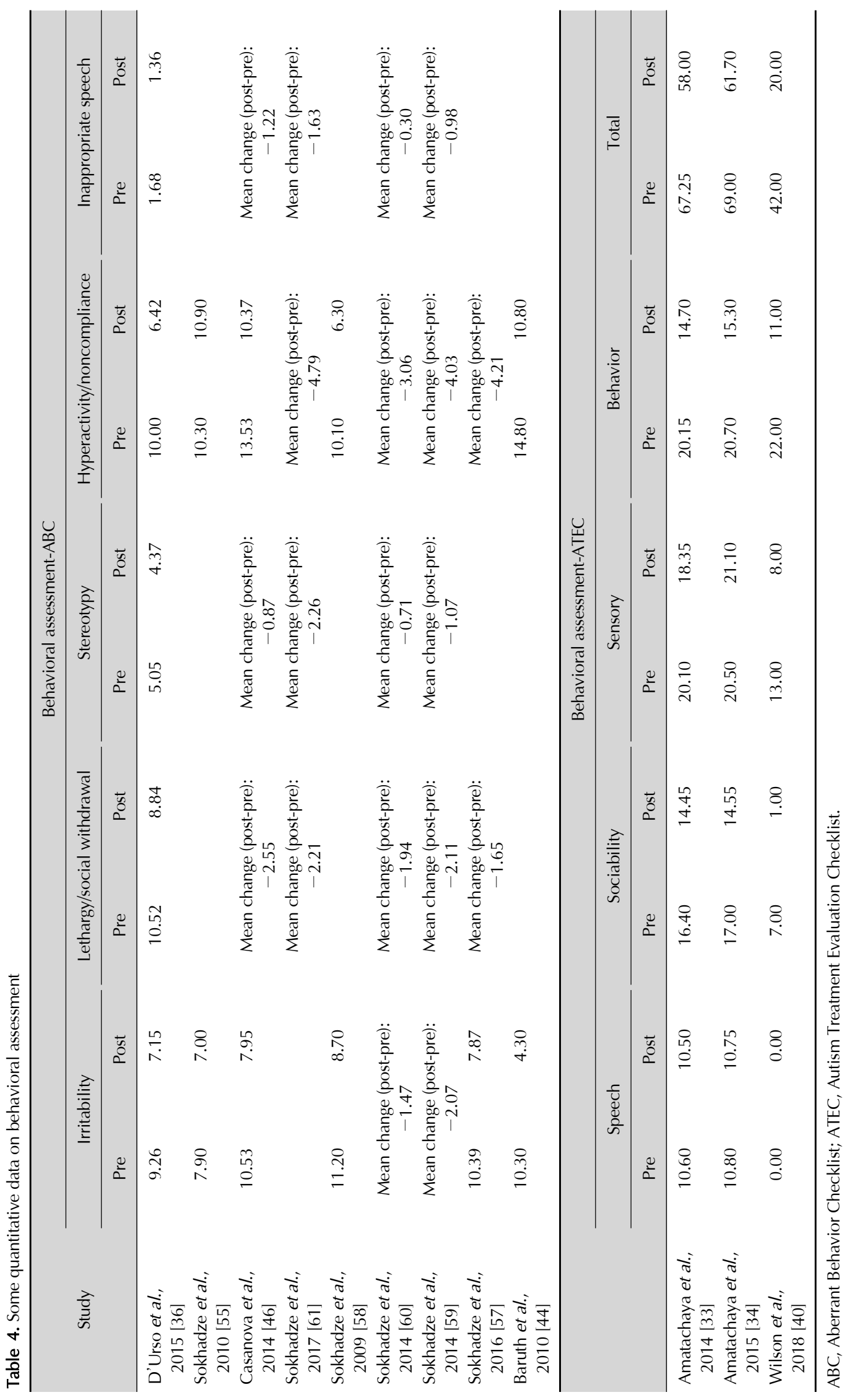




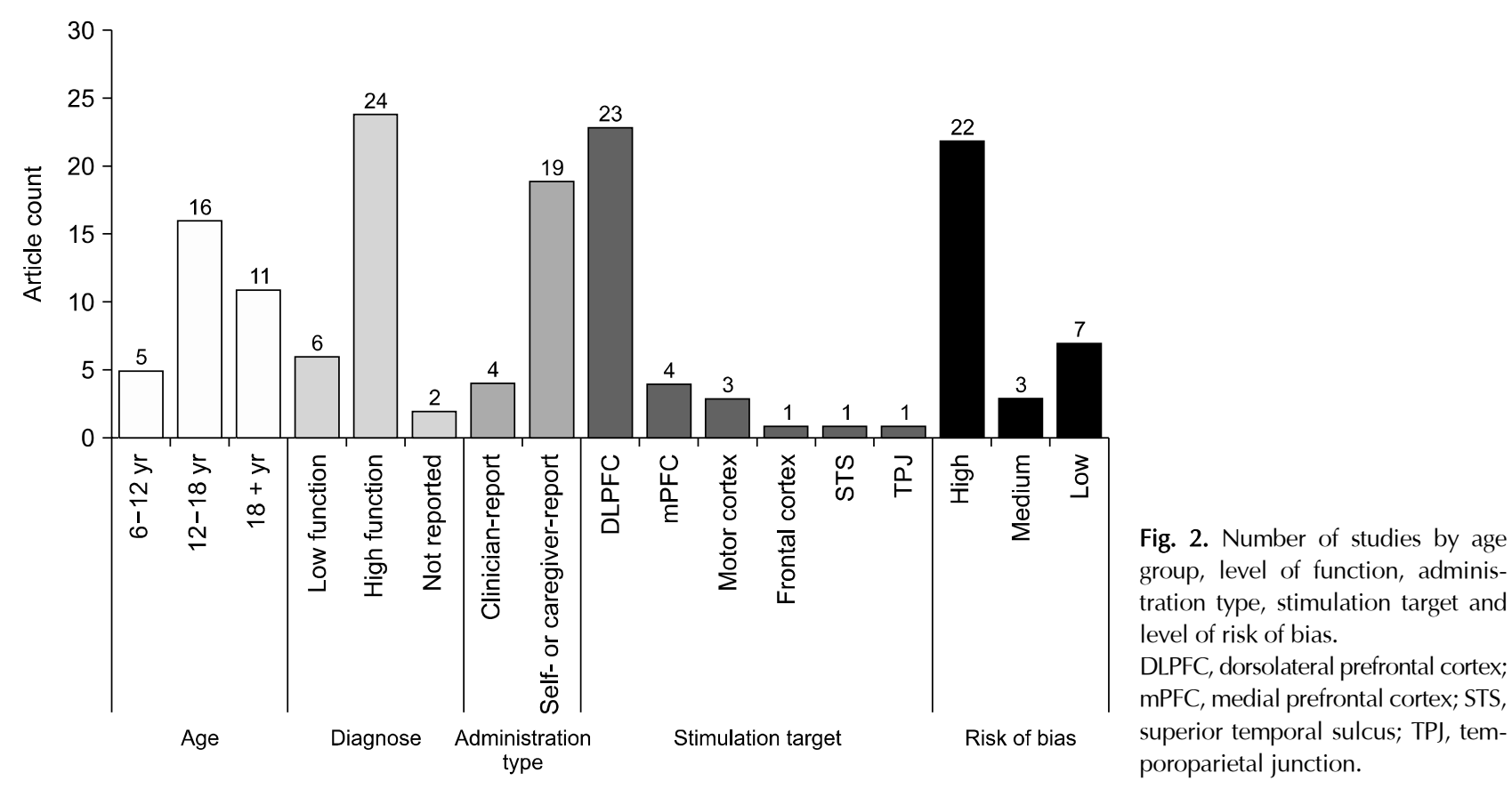

\section{COGNITIVE EFFECTS}

Eighteen studies evaluated the effects of NIBS on cognitive function in patients with ASD; fifteen of which used rTMS and the 3 remaining studies used tDCS. Of these eighteen studies, twelve were controlled studies, four were non-controlled studies and two were case reports. Among the tDCS studies, the n-back test and the brief test of attention, the bilingual aphasia test, and motor skill planning tests were administered to assess the working memory capacity and divided attention in a verbal-linguistic system, syntax acquisition, and feasibility of tDCS as a rehabilitation technique for ASD children, respectively. They found enhanced working memory in adult ASDs, improved language function both in vocabulary and syntax, and long-lasting enhancement of motor planning in ASD children after tDCS interventions over the left or bilateral DLPFC.

On the other hand, most rTMS studies assessed performance on an oddball-type task in terms of reaction time, commission and omission errors (i.e., responding to non-targets and failing to respond to the targets, respectively), and accuracy of responses or total number of errors. The oddball paradigm involves a rapid presentation of a series of repetitive standard stimuli disrupted by a deviant stimulus and requires a response from the subjects (such as pressing a button) when deviants appear. Various dimensions of executive control are involved in this task including working memory, attention control and response inhibition. All rTMS studies using oddball paradigm applied low frequency rTMS to the DLPFC bilaterally, with the exception of two studies that stimulated only the left DLPFC. However, their cognitive results are similar, with minor differences. They reported no significant change in oddball reaction time after rTMS intervention in ASD. Some of them reported no change in error rates in oddball tasks after rTMS, and some found significant decreases in omission, commission and total error rates after rTMS. Furthermore, four other studies used other neuropsychological tests to evaluate the cognitive effects of rTMS on ASD patients. They administered the Boston Naming Test, a sequential button-pressing task, and mentalizing tasks. One study reported increased response latency (worse performance) following low frequency rTMS to the left pars opercularis and decreased response latency (better performance) following rTMS to the left pars triangularis in comparison to the sham condition, and the others reported no improvements in reaction time, movement time or in mentalizing measures following stimulation. Two other studies utilized the iTBS protocol to affect cognitive functions in ASD patients. They administered Wisconsin Card Sorting Test, Conner's Continuous Performance Test, and Stroop test, and found improvements in reaction times and perseverative errors following 
DLPFC iTBS. Panerai et al. [54] designed multi-level trials to evaluate the feasibility of rTMS for enhancing eye-hand integration ability, which was assessed by the Psychoeducational Profile-Revised, in low function patients with ASD. For this purpose, they first examined different stimulation targets (right and left premotor cortex) and parameters (sham, $1 \mathrm{~Hz}$ rTMS and $8 \mathrm{~Hz}$ rTMS). They reported a significant main effect on the patients' performance following single-session as well as multisession $8 \mathrm{~Hz}$ rTMS to the left premotor cortex compared to $1 \mathrm{~Hz}$ and sham stimulation. However, they found that $8 \mathrm{~Hz}$ rTMS could not result in long-lasting effects as a standalone treatment, while a combination of $8 \mathrm{~Hz}$ rTMS and an eye-hand integration training program resulted in long-lasting effects on the patients' performance at four weeks follow-up when compared to the training program alone or the rTMS alone. In a case report, Avirame et al. [43] assessed the Mindstreams battery and Cambridge Mindreading battery following multisession $5 \mathrm{~Hz}$ rTMS to the mPFC bilaterally, and reported better performance in terms of attention, speed processing and executive functions after stimulation.

\section{BEHAVIORAL EFFECTS}

Twenty two studies evaluated the effects of NIBS on ASD behavioral symptoms; 18 of which used rTMS and the 4 remaining studies used tDCS. Of these 22 studies, 10 were controlled studies, 8 were non-controlled studies and 4 were case reports. In these studies, different questionnaires were administered to measure stimulation effects on several dimensions of behavioral symptoms in ASD including Aberrant Behavior Checklist (ABC; a behavior rating scale for the evaluation of treatment effects on mentally retarded subjects) [65], Repetitive Behavior Scale-Revised (RBS-R; a quantitative continuous measure of the breadth of repetitive behaviors) [66], Social Responsiveness Scale (SRS; a continuous measure of social ability) [67], Autism Treatment Evaluation Checklist (ATEC; an assessment tool to evaluate effectiveness of treatments over time based on autistic symptoms) [68], Autism Spectrum Quotient (AQ; a self-report questionnaire to measure severity of autistic traits) [69], Ritvo Autism-Aspergers Diagnostic Scale (RAADS; a screening instrument to identify autistic traits) [70], Childhood Autism Rating Scale (CARS; a clinical rating scale to rate items indicative of ASD) [71], Yale-Brown Obsessive-
Compulsive Scale (Y-BOCS; a semi-structured interview to measure the severity of obsessive and compulsive behaviors) [72], Autism Diagnostic Interview-Revised (ADI-R; an empirical algorithm only for the diagnosis of strict autism) [73], Interpersonal Reactivity Index (IRI; an assessment tool for the multi-dimensional measurement of empathy) [74], Children's Global Assessment Scale (CGAS; a numerical scale to measure the general functioning of youths with mental health problems) [75] and Clinical Global Impression (CGl; a rating scale to measure severity of symptoms, treatment response and the effectiveness of treatments in intervention studies on patients with mental disorders) [76]. The two most common questionnaires employed in this domain were the $A B C$ and RBS-R. Gómez et al. [64] applied both tDCS and rTMS to the left DLPFC and found a significant reduction in the total scores of ABC, ADI-R, ATEC and CGI one month after the NIBS intervention.

In the tDCS studies, all but one study, that targeted the right temporoparietal junction, stimulated the left DLPFC. All tDCS studies reported significant improvements in symptoms and behavioral problems of ASD patients and patients' functioning, especially in sociability, health/behavioral and hyperactivity/non-compliance subscales after intervention. In rTMS studies, most trials delivered multisession low frequency rTMS with stimulus frequencies of either $0.5 \mathrm{~Hz}$ or $1 \mathrm{~Hz}$, with the exception of Abujadi et al. [41] and Ni et al. [52], who used the iTBS protocol, and Anninos et al. [42], in whose work the pT-TMS protocol with stimulus frequencies of $8-13 \mathrm{~Hz}$ was used. These trials reported significant improvements in sociability, hyperactivity/noncompliance, irritability, repetitive and compulsive behaviors of ASD patients after multisession rTMS to the DLPFC bilaterally. However, using multisession rTMS to the left DLPFC, only improvement in repetitive behavior of patients has reported. Niederhofer [53] also found improvement in the irritability and stereotypy subscales of $\mathrm{ABC}$ in an adult ASD sample following a low frequency rTMS to the SMA. Furthermore, sociability has been improved in ASD using $5 \mathrm{~Hz}$ rTMS to the mPFC bilaterally. In a non-controlled design using multisession iTBS to the right DLPFC, Abujadi et al. [41] found a reduction in mean overall compulsive behaviors as well as in mean RBS-R scores, while Ni et al. [52] reported post-treatment improvements in compulsive behaviors following single-session iTBS to 
the pSTS, but not to the DLPFC, bilaterally. Anninos et al. [42] assessed social behaviors and intellectual disability in ASD children after their pT-TMS protocol. They found minor to major improvements in the active-rTMS group compared to the sham-rTMS group.

\section{BIOLOGICAL EFFECTS}

Sixteen studies assessed the effects of NIBS on physiology and neurophysiology in ASD patients using electroencephalogram (EEG), event-related potentials, bloodoxygen-level dependent (BOLD) activity, cortico-spinal excitability, electrocardiogram, heart rate variability (HRV) and skin conductance level (SCL) analysis; ten of which were controlled studies, and the six remaining were non-controlled studies. Van Steenburgh et al. [39] measured the correlations and anti-correlations of BOLD signal to assess the connectivity between the task-positive network and the default mode network in adults with ASD when simultaneously receiving tDCS over the DLPFC bilaterally and solving working memory problems. They observed that anodal tDCS over the right or left DLPFC resulted in increased functional connectivity between the posterior cingulate and mPFC. Amatachaya et al. [34] found a significant increase in the peak alpha frequency (PAF) at the stimulation site following anodal tDCS to the left DLPFC, and reported that this PAF increase was significantly correlated with improvements in behavioral symptoms of ASD patients. Desarkar et al. [63] found lessened long term depression and long term potentiation-like neuroplasticity following high frequency rTMS in active-rTMS compared to sham-rTMS. Casanova et al. [46] reported increased R-R cardiointervals and high frequency (HF) component of HRV as well as decreased low frequency (LF) component of HRV, LF/HF ratio of HRV and SCL over 18 sessions of rTMS to the DLPFC bilaterally in ASD children. These findings were repeated and confirmed later in two open-label studies conducted by Wang et al. [62] and Sokhadze et al. [61]. Enticott et al. [50] assessed different components of the movement-related cortical potentials (MRCPs) measured by EEG and the motor-evoked potentials measured by electromyography when ASD patients performed a button board task following a single-session low frequency rTMS to the SMA and left PMC. They found an improvement in the gradient of the early component of MRCPs after SMA stimulation in the active-rTMS group compared to the sham-rTMS group. Also, PMC stimulation resulted in an improvement in the gradient of the late component. They reported no rTMS impact on the motor-evoked potentials. Gómez et al. [64] assessed brain functional connectivity as well as the P300 component by a passive oddball paradigm following NIBS to the left DLPFC in ASD children. They found an increase in functional connectivity of the brain, especially for alpha, beta and gamma frequency bands, with most significant changes caused by the rTMS technique. Also, they reported a significantly shorter latency of P300 with no changes in its amplitude after intervention. Baruth et al. [44] reported increased evoked gamma responses to targets in an oddball-type task in all regions of the brain as a result of rTMS intervention to the left and right DLPFC.

Furthermore, Sokhadze and his colleagues assessed the effects of rTMS on different ERP components in their studies. All these studies applied multisession low frequency rTMS with stimulus frequencies of either $0.5 \mathrm{~Hz}$ or $1 \mathrm{~Hz}$ to the DLPFC bilaterally, with the exception of two of their studies that used a unilateral protocol to stimulate the left DLPFC. The ERP components for the anterior regions of the brain were P50, P200, P3a (P300), N100 and N200; and for the posterior regions of the brain they were P50, P200, P3b (P300), N100 and N200. Error-related positivity $(\mathrm{Pe})$ and error-related negativity (ERN), as response-locked variables, were also studied following rTMS. In EEG, the P50 is an ERP component appearing about $40-80 \mathrm{~ms}$ after the presentation of an auditory stimulus. It is extracted to measure sensory gating or the ability of the brain to selectively process sensory stimuli. Sokhadze et al. [55] reported increased amplitude of the frontal and centro-parietal P50 to targets as well as decreased amplitude of the parieto-occipital P50 to novel distracters following rTMS to the left DLPFC. Also, they found increased latency of the frontal P50 to targets post-rTMS. N100 is a large negative-going ERP component appearing about $80-120 \mathrm{~ms}$ after the presentation of a stimulus, and it is involved in perception and the person's arousal. Sokhadze and his colleagues reported decreased amplitude and prolonged latency of the frontal N100 to non-targets after rTMS intervention in their studies. P100 is a positive-going ERP component, which can be modulated by attention, occurring approximately $80-130$ ms after the onset of a visual stimulus. Sokhadze 
research group reported increased amplitude and latency of the centro-parietal P100 to targets as well as its decreased latency to non-target stimuli, post-rTMS. P200 is a positive-going ERP component occurring approximately $150-275 \mathrm{~ms}$ after the onset of a visual stimulus. It is thought that P200 is involved in higher order perceptual processing, which may compare sensory inputs with stored memory. Sokhadze research group reported prolonged latency of the coentro-parietal P200 to targets after rTMS treatment. N200 is a negative-going wave, appearing about $200-350 \mathrm{~ms}$ after the presentation of a stimulus. Previous studies have used this component for mismatch detection, language researches and for assessment of executive cognitive control functions. Sokhadze research group reported reduced latency of the frontal and parietal N200 to novel distracters and targets, as well as decreased amplitude of the frontal N200 to both targets and non-targets post-rTMS. However, they found prolonged latency of the frontal N200 to targets, prolonged latency of the parietal N200 to non-targets and decreased amplitude of the parietal N200 in some researches. P300 is a positive-going wave, appearing about 250 - $500 \mathrm{~ms}$ after the presentation of a stimulus. It is evoked in a process of decision making and is composed of two subcomponents: P3a and P3b. P3a has been shown to be associated with brain functions related to the processing of novelty and the engagement of attention, and P3b may be extracted to assess cognitive workload during a task. From the results reported by the Sokhadze research group, it can be concluded that the amplitude and latency of the P3a are reduced to non-targets post-rTMS. Moreover, they reported decreased amplitude and latency of the P3b to non-targets and prolonged latency of the P3b to targets after rTMS intervention. ERN is a sharp negative-going ERP component appearing about 40-150 ms after an incorrect motor response begins, even when the subject is not explicitly conscious of making the error. The ERN component is followed by a positive-going wave, known as the Pe. The Pe is basically associated with conscious sensations and perception of the error. Sokhadze research group reported increased amplitude and decreased latency of the ERN during commission errors and no significant changes for Pe, post-rTMS. They also reported decreased power of evoked gamma oscillations for non-targets after rTMS treatment in their ASD samples.

\section{DISCUSSION}

In this systematic review, we investigated the existing evidence on the use of NIBS, including tDCS and rTMS, to treat ASD. For this purpose, we inspected 32 original studies of the available literature reporting the effects of NIBS techniques on ASD-related behavioral, cognitive and neurophysiological dysfunctions. In general, there is a very large heterogeneity and variability between studies in terms of patients' profiles, study designs, schedules and parameters of stimulation and so on that makes drawing any conclusions about the promise of these techniques difficult and even impossible. However, most of these studies have reported positive effects of NIBS methods, regardless of variables such as age, sex, severity of disorder, design, type and area of stimulation. These trials stimulated different areas of the brain based on various hypotheses about the neural impairments in ASD. Prefrontal cortex (PFC), especially DLPFC, is a main target region for stimulation. Growing evidence highlights the biological basis of ASD. Early symptoms appear before the age of three. This suggests that neurochemical and neuroanatomical mechanisms are the underlying pathophysiology of ASD occurring in the early development of the central nervous system. Neuroimaging studies have demonstrated PFC impairments that result in mentalizing and social related deficits in ASD [77]. In fact, the main purpose of most trials is to balance and normalize the cortical excitation to inhibition ratio and to improve the long-range cortical connectivity (i.e., anterior-posterior interconnection). This comes from the hypothesis that abnormal cortical minicolumnar organization may lead to impairments in inhibitory GABAergic fiber projections, which result in sensory disorders as well as in the occurrence of epilepsy in ASD [14]. Several studies have mentioned that autism could be associated with dopaminergic dysfunction and have assumed that dopamine imbalances in some brain areas may result in autistic behaviors. In fact, patients with ASD have shown changes in the mesocorticolimbic dopaminergic signaling pathway, including decreased dopamine release in the PFC $[78,79]$. A recent study has also shown reduced glutamate concentration in the striatum that was associated with sociability in ASD [80]. Due to their effects on neurotransmitter systems in different brain areas, various NIBS protocols can result in regulation of brain function in ASD to some extent. Bifrontal tDCS and 
high frequency rTMS to the DLPFC have proved to increase dopamine releases and levels in different brain regions, including striatum and caudate [26]. Furthermore, motor areas were targeted based on the hypothesis that deficits in these areas, such as PMC and SMA dysfunctions, may contribute to problems in motor functions, especially those related to the preparation of movement, in ASD [50]. According to neuroimaging studies that reported reduced regional blood flow in the bilateral superior temporal sulcus and its association with ASD symptoms $[6,52]$, one trial selected pSTS as a potential target for stimulation. However, no significant improvement in the patients' function was reported after this trial, which could be due to its single-session stimulation protocol. Language-related neural networks (Broca's area) were also targeted to modulate naming skills in ASD, suggesting that the language-related neural network in ASD may be different from neurotypical subjects [51].

tDCS and rTMS are one of the most promising noninvasive neuroregulation approaches for altering cortical excitability and inducing functional reorganization of the human brain even for a short-term. It should be noted that the direction and magnitude of neuroplasticity evoked by tDCS or rTMS depend on the stimulation parameters (stimulation site, intensity, frequency, montage, number of sessions and so on) and the functional condition of the targeted region [18]. However, the clinical utility of NIBS in ASD is questionable and there are still critical challenges that limit the use of NIBS techniques for ASD treatment. Most studies were case-report and open-label trials with low levels of evidence for clinical application. Moreover, most controlled trials were conducted using a waiting-list group or healthy individuals instead of sham stimulation as the control/comparison group. Also, more than $80 \%$ of studies were administered by self-report or caregiver-report (mostly based on parent reports) approaches. Consequently, their results are likely to be affected by placebo-effects. Lack of sufficient blinding and random allocation is also a critical problem in the design of most existing trials that may have considerable impacts on the observed findings. A good trial should minimize the variability of the assessment and provide an unbiased assessment of the intervention by preventing confounding from other known or unknown factors. Randomization removes selection bias, generates comparable intervention groups and provides a basis for statistical analysis. How- ever, randomization alone is not enough, and blinding is another crucial methodological feature of RCTs, which minimizes ascertainment and performance bias after randomization. As tDCS and rTMS devices often have a blinding feature, it is strongly recommended that future studies should not overlook the double blinding feature. Therefore, to gain more insight into the long-term effects of NIBS on ASD, we need well-designed longitudinal experimental protocols with an adequate follow-up period after the treatment course, which has not been met well in existing studies.

Although these trials tested NIBS techniques almost in the entire autistic spectrum, less than $20 \%$ of them enrolled low-functioning patients in the study. Almost all of these studies used case-report or open-label designs and they have a high risk of bias (according to the Cochrane guideline, risk of bias is defined as the risk of a systematic error in the design and conduct of a study, as well as in its results or inferences) (except for the Panerai study that did not measure behavioral outcomes). Therefore, it might be better to limit the positive results of the trials to high-function autism. This limitation should be considered for future studies by researchers in this field. In addition, half of the studies were conducted on adolescent patients (1218-year-olds), one-third on adult patients ( $>18$-year-olds), and about one-sixth on autistic children (6-12-year-olds). Therefore, the obtained results can hardly be considered valid for children from 6 to 12 years of age, and they cannot be generalized to preschool children.

Furthermore, there are still concerns about safety, tolerability and ethical issues. More than half of the existing studies have not reported side effects, and the rest have often failed to use valid questionnaires to assess side effects. Therefore, future studies should utilize a standard side effect questionnaire to evaluate the tolerability and feasibility of NIBS procedures, especially in younger and lower functioning patients with autism. In addition, it is strongly recommended that the medical history, current medication or psychotherapy and risk-benefit ratio should be carefully assessed. Given that NIBS not only affects the stimulation site, but also modulates other brain regions, future studies should carefully monitor the behavioral and physiological domains of patients in the long-term follow-up periods for any potential NIBS-induced negative effects.

Researchers should select NIBS techniques (tDCS or 
rTMS) and stimulation protocols (excitatory or inhibitory) based on the current understanding of autism pathophysiology and neuropathology. This helps to learn more about the pathogenesis of autism and, also, to reduce heterogeneity among studies. In this regard, it is suggested that tDCS trials use cathodal protocols (inhibitory) for DLPFC stimulation and anodal protocols (excitatory) for mPFC stimulation as rTMS studies that utilize suppressive protocols for DLPFC and facilitatory protocols for $\mathrm{mPFC}$ stimulation. In general, the tDCS procedure has been used much less than the rTMS; thus, more studies should be conducted using tDCS in the future to assess its feasibility. Because of its neuroregulatory properties and advantages, such as ease of use, home use, portability and low cost, tDCS is an interesting tool whose efficacy is proven in some other diseases and disorders and it can be used in combination with other treatments. Therefore, it can be very useful to investigate the NIBS techniques as complementary therapies along with standard pharmaceutical treatments, effective behavioral teaching, especially in young patients, and new medications. This is because brain stimulation (with rational and accurate protocols) may strengthen the mechanism of action of drugs that target the pathophysiology of autism. Given the abnormalities of high frequency waves (particularly gamma oscillations) of the electrical activity of the brain and their association with the problems in the synchronization and connectivity of neural assemblies in autism [57], it is suggested to use tACS with appropriate frequencies in future studies.

Given that we did not consider any limitations regarding the study design, and the age or sex of participants, these results must be interpreted with caution regarding the effect of confounding factors in the systematic review. This issue is a major limitation of this study. However, we tried to include all relevant researches in this work to draw a comprehensive picture of the current condition in this new therapeutic field in order to review its findings, and describe its strengths and weaknesses.

Existing evidence demonstrates that NIBS methods could be helpful for treating some dimensions of ASD such as repetitive and stereotyped behavior, sociability or some aspects of executive and cognitive functions. However, such evidence should be regarded with care because of the quality of the original researches and serious publication bias as well as the heterogeneity of data.
In addition, it should be noted that the NIBS procedure has inherent practical constraints. For example, autistic people with epilepsy should be excluded from this therapeutic approach; so, a large population of patients with ASD cannot benefit from the NIBS treatments. Furthermore, we still have no idea about the durability of the NIBS-induced positive effects on ASD. Also, very little is known about the most effective stimulation parameters, brain targets, and treatment schedules. Therefore, further randomized, double-blind, sham-controlled trials with appropriate follow-up periods should be designed to assess the efficacy and effectiveness of NIBS methods for ASD treatment. In conclusion, available evidence should be considered as insufficient and preliminary to support the short-term and long-term efficacy of NIBS to treat autistic people.

\section{Acknowledgments}

We acknowledge the financial support of Tehran University of Medical Sciences through a grant from Psychiatry and Psychology Research Center (grant number 39074). The authors also wish to thank Fatemeh Daftari (Department of English, Tehran University) for language editing during the drafting of this paper.

\section{Conflicts of Interest}

No potential conflict of interest relevant to this article was reported.

\section{Author Contributions}

Search strategy and data extraction: Ali Khaleghi, Safa Rafiei Vand, and Hadi Zarafshan. Writing - original draft: Ali Khaleghi. All authors were involved in the design of the study. All authors read and approved the final manuscript.

\section{ORCID}

Ali Khaleghi https://orcid.org/0000-0002-9035-7075

Hadi Zarafshan https://orcid.org/0000-0002-3188-5046

Safa Rafiei Vand https://orcid.org/0000-0002-4749-9721 Mohammad Reza Mohammadi

https://orcid.org/0000-0002-7061-5938

\section{REFERENCES}

1. Zarafshan H, Mohammadi MR, Motevalian SA, Abolhassani F, Khaleghi A, Sharifi V. Autism research in Iran: a sciento- 
metric study. Iran J Psychiatry Behav Sci 2017;11:e7350.

2. American Psychiatric Association. Diagnostic and statistical manual of mental disorders: DSM-5. 5th ed. Arlington: American Psychiatric Association;2013.

3. Mohammadi MR, Ahmadi N, Khaleghi A, Mostafavi SA, Kamali K, Rahgozar M, et al. Prevalence and correlates of psychiatric disorders in a national survey of Iranian children and adolescents. Iran J Psychiatry 2019;14:1-15.

4. Khaleghi A, Mohammadi MR, Zandifar A, Ahmadi N, Alavi SS, Ahmadi A, et al. Epidemiology of psychiatric disorders in children and adolescents; in Tehran, 2017. Asian J Psychiatr 2018;37:146-153.

5. Mohammadi MR, Akhondzadeh S. Autism spectrum disorders: etiology and pharmacotherapy. Curr Drug Ther 2007; 2:97-103.

6. Ohnishi $\mathrm{T}$, Matsuda $\mathrm{H}$, Hashimoto $\mathrm{T}$, Kunihiro $\mathrm{T}$, Nishikawa $\mathrm{M}$, Uema $\mathrm{T}$, et al. Abnormal regional cerebral blood flow in childhood autism. Brain 2000;123(Pt 9):1838-1844.

7. Nickl-Jockschat T, Habel U, Michel TM, Manning J, Laird AR, Fox PT, et al. Brain structure anomalies in autism spectrum disorder--a meta-analysis of VBM studies using anatomic likelihood estimation. Version 2. Hum Brain Mapp 2012;33: 1470-1489.

8. LeBlanc JJ, Fagiolini M. Autism: a "critical period" disorder? Neural Plast 2011;2011:921680.

9. Baruth JM, Wall CA, Patterson MC, Port JD. Proton magnetic resonance spectroscopy as a probe into the pathophysiology of autism spectrum disorders (ASD): a review. Autism Res 2013;6:119-133.

10. Herbert MR, Harris GJ, Adrien KT, Ziegler DA, Makris N, Kennedy DN, et al. Abnormal asymmetry in language association cortex in autism. Ann Neurol 2002;52:588-596.

11. Lindell AK, Hudry K. Atypicalities in cortical structure, handedness, and functional lateralization for language in autism spectrum disorders. Neuropsychol Rev 2013;23:257-270.

12. Persico AM, Bourgeron T. Searching for ways out of the autism maze: genetic, epigenetic and environmental clues. Trends Neurosci 2006;29:349-358.

13. Hamilton AF. Emulation and mimicry for social interaction: a theoretical approach to imitation in autism. Q J Exp Psychol (Hove) 2008;61:101-115.

14. Casanova MF, van Kooten IA, Switala AE, van Engeland $H$, Heinsen $\mathrm{H}$, Steinbusch HW, et al. Minicolumnar abnormalities in autism. Acta Neuropathol 2006;112:287-303.

15. Demirtas-Tatlidede A, Vahabzadeh-Hagh AM, Pascual-Leone A. Can noninvasive brain stimulation enhance cognition in neuropsychiatric disorders? Neuropharmacology 2013;64: 566-578.

16. Kuo MF, Paulus W, Nitsche MA. Therapeutic effects of non-invasive brain stimulation with direct currents (tDCS) in neuropsychiatric diseases. Neuroimage 2014;85 Pt 3:948960.

17. Nitsche MA, Cohen LG, Wassermann EM, Priori A, Lang N,
Antal A, et al. Transcranial direct current stimulation: state of the art 2008. Brain Stimul 2008;1:206-223.

18. Mostafavi SA, Khaleghi A, Mohammadi MR, Akhondzadeh S. Is transcranial direct current stimulation an effective modality in reducing food craving? A systematic review and metaanalysis. Nutr Neurosci 2020;23:55-67.

19. Boggio PS, Nunes A, Rigonatti SP, Nitsche MA, Pascual-Leone A, Fregni F. Repeated sessions of noninvasive brain DC stimulation is associated with motor function improvement in stroke patients. Restor Neurol Neurosci 2007;25:123-129.

20. Nitsche MA, Liebetanz D, Antal A, Lang N, Tergau F, Paulus W. Modulation of cortical excitability by weak direct current stimulation--technical, safety and functional aspects. Suppl Clin Neurophysiol 2003;56:255-276.

21. Liebetanz D, Nitsche MA, Tergau F, Paulus W. Pharmacological approach to the mechanisms of transcranial DC-stimulationinduced after-effects of human motor cortex excitability. Brain 2002;125(Pt 10):2238-2247.

22. Hallett M. Transcranial magnetic stimulation: a primer. Neuron 2007;55:187-199.

23. Wagner T, Valero-Cabre A, Pascual-Leone A. Noninvasive human brain stimulation. Annu Rev Biomed Eng 2007;9:527565.

24. Hoogendam JM, Ramakers GM, Di Lazzaro V. Physiology of repetitive transcranial magnetic stimulation of the human brain. Brain Stimul 2010;3:95-118.

25. Huang YZ, Edwards MJ, Rounis E, Bhatia KP, Rothwell JC. Theta burst stimulation of the human motor cortex. Neuron 2005;45:201-206.

26. Mostafavi SA, Khaleghi A, Mohammadi MR. Noninvasive brain stimulation in alcohol craving: a systematic review and meta-analysis. Prog Neuropsychopharmacol Biol Psychiatry 2020;101:109938.

27. Khaleghi A, Pirzad Jahromi G, Zarafshan H, Mostafavi SA, Mohammadi MR. Effects of transcranial direct current stimulation of prefrontal cortex on risk-taking behavior. Psychiatry Clin Neurosci 2020. doi: 10.1111/pcn.13025. [Epub ahead of print]

28. Rossi S, Hallett M, Rossini PM, Pascual-Leone A; Safety of TMS Consensus Group. Safety, ethical considerations, and application guidelines for the use of transcranial magnetic stimulation in clinical practice and research. Clin Neurophysiol 2009;120:2008-2039.

29. Poreisz C, Boros K, Antal A, Paulus W. Safety aspects of transcranial direct current stimulation concerning healthy subjects and patients. Brain Res Bull 2007;72:208-214.

30. Oberman LM, Enticott PG, Casanova MF, Rotenberg A, Pascual-Leone A, McCracken JT; TMS in ASD Consensus Group. Transcranial magnetic stimulation in autism spectrum disorder: challenges, promise, and roadmap for future research. Autism Res 2016;9:184-203.

31. Barahona-Corrêa JB, Velosa A, Chainho A, Lopes R, OliveiraMaia AJ. Repetitive transcranial magnetic stimulation for 
treatment of autism spectrum disorder: a systematic review and meta-analysis. Front Integr Neurosci 2018;12:27.

32. Higgins JPT, Green S. Cochrane handbook for systematic reviews of interventions 4.2.5. Chichester:John Wiley \& Sons; 2005.

33. Amatachaya A, Auvichayapat $\mathrm{N}$, Patjanasoontorn $\mathrm{N}$, Suphakunpinyo C, Ngernyam N, Aree-Uea B, et al. Effect of anodal transcranial direct current stimulation on autism: a randomized double-blind crossover trial. Behav Neurol 2014;2014:173073.

34. Amatachaya A, Jensen MP, Patjanasoontorn N, Auvichayapat $\mathrm{N}$, Suphakunpinyo $\mathrm{C}$, Janjarasjitt $\mathrm{S}$, et al. The short-term effects of transcranial direct current stimulation on electroencephalography in children with autism: a randomized crossover controlled trial. Behav Neurol 2015;2015:928631.

35. Hupfeld KE, Ketcham CJ. Behavioral effects of transcranial direct current stimulation on motor and language planning in minimally verbal children with autism spectrum disorder (ASD): feasibility, limitations and future directions. J Child Dev Disord 2016;2:21.

36. D’Urso G, Bruzzese D, Ferrucci R, Priori A, Pascotto A, Galderisi S, et al. Transcranial direct current stimulation for hyperactivity and noncompliance in autistic disorder. World J Biol Psychiatry 2015;16:361-366.

37. Schneider HD, Hopp JP. The use of the Bilingual Aphasia Test for assessment and transcranial direct current stimulation to modulate language acquisition in minimally verbal children with autism. Clin Linguist Phon 2011;25:640-654.

38. van Steenburgh J, Varvaris M, Schretlen DJ, Vannorsdall TD, Gordon B. Balanced bifrontal transcranial direct current stimulation enhances working memory in adults with high-functioning autism: a sham-controlled crossover study. Mol Autism 2017;8:40.

39. van Steenburgh JJ, Varvaris M, Vannorsdall TD, Schretlen DJ, Gordon B. Transcranial direct current stimulation enhances functional connectivity in high-functioning individuals with autism. Annals of Neurology. In: 139th Annual Meeting American Neurological Association; Oct 12-14, 2014; Baltimore. Abstract NR S118:S1.

40. Esse Wilson J, Quinn DK, Wilson JK, Garcia CM, Tesche CD. Transcranial direct current stimulation to the right temporoparietal junction for social functioning in autism spectrum disorder: a case report. J ECT 2018;34:e10-e13.

41. Abujadi C, Croarkin PE, Bellini BB, Brentani H, Marcolin MA. Intermittent theta-burst transcranial magnetic stimulation for autism spectrum disorder: an open-label pilot study. Version 2. Braz I Psychiatry 2018;40:309-311.

42. Anninos P, Chatzimichael A, Adamopoulos A, Kotini A, Tsagas N. A combined study of MEG and pico-Tesla TMS on children with autism disorder. J Integr Neurosci 2016;15:497513.

43. Avirame K, Stehberg J, Todder D. Enhanced cognition and emotional recognition, and reduced obsessive compulsive symptoms in two adults with high-functioning autism as a result of deep transcranial magnetic stimulation (dTMS): a case report. Neurocase 2017;23:187-192.

44. Baruth JM, Casanova MF, El-Baz A, Horrell T, Mathai G, Sears $\mathrm{L}$, et al. Low-frequency repetitive transcranial magnetic stimulation (rTMS) modulates evoked-gamma frequency oscillations in autism spectrum disorder (ASD). J Neurother 2010; 14:179-194.

45. Casanova MF, Baruth JM, El-Baz A, Tasman A, Sears L, Sokhadze E. Repetitive transcranial magnetic stimulation (rTMS) modulates event-related potential (ERP) indices of attention in autism. Trans/ Neurosci 2012;3:170-180.

46. Casanova MF, Hensley MK, Sokhadze EM, El-Baz AS, Wang $\mathrm{Y}$, Li X, et al. Effects of weekly low-frequency rTMS on autonomic measures in children with autism spectrum disorder. Front Hum Neurosci 2014;8:851.

47. Cristancho P, Akkineni K, Constantino JN, Carter AR, O'Reardon JP. Transcranial magnetic stimulation in a 15-year-old patient with autism and comorbid depression. J ECT 2014;30:e46e47.

48. Enticott PG, Fitzgibbon BM, Kennedy HA, Arnold SL, Elliot D, Peachey A, et al. A double-blind, randomized trial of deep repetitive transcranial magnetic stimulation (rTMS) for autism spectrum disorder. Brain Stimul 2014;7:206-211.

49. Enticott PG, Kennedy HA, Zangen A, Fitzgerald PB. Deep repetitive transcranial magnetic stimulation associated with improved social functioning in a young woman with an autism spectrum disorder. J ECT 2011;27:41-43.

50. Enticott PG, Rinehart NJ, Tonge BJ, Bradshaw JL, Fitzgerald PB. Repetitive transcranial magnetic stimulation (rTMS) improves movement-related cortical potentials in autism spectrum disorders. Brain Stimul 2012;5:30-37.

51. Fecteau S, Agosta S, Oberman L, Pascual-Leone A. Brain stimulation over Broca's area differentially modulates naming skills in neurotypical adults and individuals with Asperger's syndrome. Eur J Neurosci 2011;34:158-164.

52. Ni HC, Hung J, Wu CT, Wu YY, Chang CJ, Chen RS, et al. The impact of single session intermittent theta-burst stimulation over the dorsolateral prefrontal cortex and posterior superior temporal sulcus on adults with autism spectrum disorder. Front Neurosci 2017;11:255.

53. Niederhofer $\mathrm{H}$. Effectiveness of the repetitive transcranical magnetic stimulation (rTMS) of $1 \mathrm{~Hz}$ for autism. Clin Neuropsychiatry 2012;9:107.

54. Panerai S, Tasca D, Lanuzza B, Trubia G, Ferri R, Musso S, et al. Effects of repetitive transcranial magnetic stimulation in performing eye-hand integration tasks: four preliminary studies with children showing low-functioning autism. Autism 2014;18:638-650.

55. Sokhadze E, Baruth J, Tasman A, Mansoor M, Ramaswamy R, Sears $\mathrm{L}$, et al. Low-frequency repetitive transcranial magnetic stimulation (rTMS) affects event-related potential measures of novelty processing in autism. Appl Psychophysiol Biofeedback 
2010;35:147-161.

56. Sokhadze EM, Baruth JM, Sears L, Sokhadze GE, El-Baz AS, Casanova MF. Prefrontal neuromodulation using rTMS improves error monitoring and correction function in autism. Appl Psychophysiol Biofeedback 2012;37:91-102.

57. Sokhadze EM, Casanova MF, El-Baz AS, Farag HE, Li X, Wang Y. TMS-based neuromodulation of evoked and induced gamma oscillations and event-related potentials in children with autism. NeuroRegulation 2016;3:101-126.

58. Sokhadze EM, El-Baz A, Baruth J, Mathai G, Sears L, Casanova MF. Effects of low frequency repetitive transcranial magnetic stimulation (rTMS) on gamma frequency oscillations and event-related potentials during processing of illusory figures in autism. J Autism Dev Disord 2009;39:619-634.

59. Sokhadze EM, El-Baz AS, Sears LL, Opris I, Casanova MF. rTMS neuromodulation improves electrocortical functional measures of information processing and behavioral responses in autism. Front Syst Neurosci 2014;8:134.

60. Sokhadze EM, El-Baz AS, Tasman A, Sears LL, Wang Y, Lamina EV, et al. Neuromodulation integrating rTMS and neurofeedback for the treatment of autism spectrum disorder: an exploratory study. Appl Psychophysiol Biofeedback 2014;39: 237-257.

61. Sokhadze G, Casanova MF, Kelly D, Casanova E, Russell B, Sokhadze EM. Neuromodulation based on rTMS affects behavioral measures and autonomic nervous system activity in children with autism. NeuroRegulation 2017;4:65-78.

62. Wang Y, Hensley MK, Tasman A, Sears L, Casanova MF, Sokhadze EM. Heart rate variability and skin conductance during repetitive TMS course in children with autism. Appl Psychophysiol Biofeedback 2016;41:47-60.

63. Desarkar P, Rajji TK, Ameis SH, Lai MC, Blumberger D, Daskalakis ZJ. Assessing and stabilizing aberrant neuroplasticity in autism spectrum disorder using transcranial magnetic stimulation: preliminary findings from a proof-of-principle study. Brain Stimulation 2017;10:389.

64. Gómez L, Vidal B, Maragoto C, Morales LM, Berrillo S, Vera Cuesta $\mathrm{H}$, et al. Non-invasive brain stimulation for children with autism spectrum disorders: a short-term outcome study. Behav Sci (Basel) 2017;7:63.

65. Aman MG, Burrow WH, Wolford PL. The Aberrant Behavior Checklist-Community: factor validity and effect of subject variables for adults in group homes. Am J Ment Retard 1995; 100:283-292.

66. Bodfish JW, Symons FW, Lewis MH. The repetitive behavior scale (Western Carolina Center research reports). Morganton (NC):Western Carolina Center; 1999.

67. Constantino JN, Gruber CP. Social responsiveness scale: SRS-2 software kit. Torrane:Western Psychological Services;
2012.

68. Geier DA, Kern JK, Geier MR. A prospective cross-sectional cohort assessment of health, physical, and behavioral problems in autism spectrum disorders. Maedica (Buchar) 2012;7: 193-200.

69. Baron-Cohen S, Wheelwright S, Skinner R, Martin J, Clubley E. The autism-spectrum quotient (AQ): evidence from Asperger syndrome/high-functioning autism, males and females, scientists and mathematicians. J Autism Dev Disord 2001;31:5-17.

70. Ritvo RA, Ritvo ER, Guthrie D, Yuwiler A, Ritvo MJ, Weisbender L. A scale to assist the diagnosis of autism and Asperger's disorder in adults (RAADS): a pilot study. J Autism Dev Disord 2008;38:213-223.

71. Rellini E, Tortolani D, Trillo S, Carbone S, Montecchi F. Childhood Autism Rating Scale (CARS) and Autism Behavior Checklist (ABC) correspondence and conflicts with DSM-IV criteria in diagnosis of autism. J Autism Dev Disord 2004;34: 703-708.

72. Goodman WK, Price LH, Rasmussen SA, Mazure C, Fleischmann RL, Hill CL, et al. The Yale-Brown Obsessive Compulsive Scale. I. Development, use, and reliability. Arch Gen Psychiatry 1989;46:1006-1011.

73. Lord C, Rutter M, Le Couteur A. Autism Diagnostic InterviewRevised: a revised version of a diagnostic interview for caregivers of individuals with possible pervasive developmental disorders. J Autism Dev Disord 1994;24:659-685.

74. Davis MH. Measuring individual differences in empathy: Evidence for a multidimensional approach. J Personal Soc Psychol 1983;44:113-126.

75. Shaffer D, Gould MS, Brasic J, Ambrosini P, Fisher P, Bird H, et al. A children's global assessment scale (CGAS). Arch Gen Psychiatry 1983;40:1228-1231.

76. Busner J, Targum SD. The clinical global impressions scale: applying a research tool in clinical practice. Psychiatry (Edgmont) 2007;4:28-37.

77. Castelli F, Frith C, Happé F, Frith U. Autism, Asperger syndrome and brain mechanisms for the attribution of mental states to animated shapes. Brain 2002;125(Pt 8):1839-1849.

78. Ernst M, Zametkin AJ, Matochik JA, Pascualvaca D, Cohen RM. Low medial prefrontal dopaminergic activity in autistic children. Lancet 1997;350:638.

79. Marotta R, Risoleo MC, Messina G, Parisi L, Carotenuto M, Vetri L, et al. The neurochemistry of autism. Brain Sci 2020; 10:163.

80. Horder J, Petrinovic MM, Mendez MA, Bruns A, Takumi T, Spooren W, et al. Glutamate and GABA in autism spectrum disorder-a translational magnetic resonance spectroscopy study in man and rodent models. Transl Psychiatry 2018;8: 106. 